Original Article

\title{
BEES (HYMENOPTERA, APOIDEA, APIFORMES) IN THE AGRICULTURAL LANDSCAPE OF BULGARIA: SPECIES DIVERSITY
}

\author{
Józef Banaszak ${ }^{1 *}$ \\ Bojana Dochkova² \\ ${ }^{1}$ Institute of Environmental Biology, Kazimierz Wielki University, 12 Ossolińskich Av., \\ 85-093 Bydgoszcz, Poland \\ 25800 Pleven, 23 San Stefano, ap. 4, Bulgaria \\ *corresponding author: lednica@ukw.edu.pl \\ Received 27 December 2012; accepted 15 January 2014
}

\begin{abstract}
A bstract
Wild bees (Apiformes) were studied in 4 crop fields and 8 refuge habitats for 2 - 5 years in agricultural landscapes in the Pleven and Plovdiv regions of Bulgaria. In total, 233 bee species were recorded. Bee forage plants visited by the honey bee and wild Apiformes are listed for each refuge habitat. Species composition is given for individual habitats, including fields of alfalfa (Medicago sativa), oilseed rape (Brassica napus), sunflower (Helianthus annuus), and radish (Raphanus sativus). Species richness and dominance structure of bee communities in the 2 regions are compared, and species responsible for significant differences are identified.
\end{abstract}

Keywords: Bulgaria, dominance structure, Hymenoptera: Apoidea: Apiformes, Pleven region, Plovdiv region, species diversity.

\section{INTRODUCTION}

Although Bulgaria lies at the borders of several zoogeographic regions, the Bulgarian fauna of Hymenoptera, including wild bees (Apiformes), is poorly studied. Among the Apiformes, bumblebees are relatively well known, mostly thanks to Pittioni's $(1938,1939)$ research on Bulgaria and the whole Balkan Peninsula, supplemented by Atanassov (1939, 1974). Other wild bees have been investigated mostly by the latter author, e.g., Halictidae (Atanassov, 1960) and Xylocopa (Atanassov, 1962a), who also published faunistic lists for the Balkan Mountains (Stara Planina) and the Petrič region (Atanassov, 1962b), and from the island of Tasos (Atanassov, 1965). In the 1970s and '80s, more attention was paid to pollinators of crop plants, especially alfalfa, and to yields of this valuable forage plant as well as human management of Megachile rotundata $\mathrm{F}$. (= M. pacifica Panz.) for pollination of alfalfa seed production fields. Most information on this subject can be found in numerous publications by Dochkova and colleagues concerning the Pleven region (e.g., Dochkova, 1981a, b, 1982a, b, 1984; Dochkova et al., 1981a, b, c, d, 1984, 1987). Alfalfa pollination and pollinators from north-eastern Bulgaria (Ruse) were also investigated by Dimitrov (1984, 1987, 1990, 1992a, b), Dimitrov et al. (1987), and Dimitrov and Dimitrova (1991a, b). Dimitrov et al. (1992) additionally studied pollinators of sunflower in the Ruse region. In the available literature, there is no information on the total number of bee species in Bulgaria, but the number is certainly high. Thus, the published literature currently includes only scanty reports on the bee fauna of this country.

Here we present data on wild bees collected in 2 types of agricultural landscapes: in the Danubian Plain (Pleven region in northern Bulgaria) and in the Upper Thracian Plain (Plovdiv region in central Bulgaria). Material was collected in 1986 - 1991, mostly along field margins and within fields of selected crop plants.

The study aims were (1) to assess the diversity and abundance of Apiformes in these 2 types of agricultural landscapes and (2) to investigate the dominance structure of pollinating insects and their phenology in these regions. 


\section{MATERIAL AND METHODS}

Field research was conducted from April/May to September/October in 1986 - 1991, but the number of years varied between regions and individual study sites, as explained in the list of study sites below. In the first year, research was initiated in late July.

The insects were caught with an aerial insect net by searching on flowers and in favorite bee nesting sites. Bee abundance was assessed once or twice a month using the transect method (Banaszak, 1980), which consists of walking along a linear transect (200 m long, $1 \mathrm{~m}$ wide) and counting or, if necessary, catching observed insects. The investigations were conducted in conditions favorable for bee activity, i.e., when air temperature was $220^{\circ} \mathrm{C}$ and the wind was not strong. Obviously, we did not catch the species that could be readily identified, such as the honey bee and some of the larger wild bees. Each sample was composed of insects noted or caught in an area of $200 \mathrm{~m}^{2}$. Bee abundance was next calculated and expressed as number of individuals per hectare. On every sampling occasion, 2 such samples were collected at each site. In further analysis, those coupled transects are considered as one sample. While counting the insects, we also recorded species of visited bee forage plants. As a result, a list of bee forage plants was compiled for individual sites.

To identify differences in the structure of wild bee communities between the study sites located in two agro-ecological landscapes (Pleven and Plovdiv), we used non-metric multidimensional scaling (NMDS). Similarity between samples was assessed on the basis of Bray-Curtis distances because of their close association with changes in community composition (Faith et al., 1987). Ordination was used for the samples where at least 5 individuals were observed, and the variables were the species represented by at least 5 individuals. The results reflected major patterns in the communities. Statistical significance of differences between communities (groups of samples) was assessed using a multi-response permutation procedure (MRPP). This method tests a hypothesis that there are no differences in species composition between study sites (Zimmerman et al., 1985; Bonner et al., 2009) and is based on the matrix of Bray-Curtis distances to compare mean dissimilarity within groups to dissimilarity between groups. The method estimates not only statistical significance but also the A statistic and is a measure of within-group homogeneity compared to random homogeneity. Its value varies from 0 (random) to 1 (all elements identical in the group). MRPP (similar to ANOSIM and ADONIS) may be used to indicate differences between groups of objects in multidimensional space (Dufrêne and Legendre, 1997; Hannon and Sisk, 2009).

To identify which species in the community are responsible for the lack of homogeneity between groups of samples, we calculated indicator values (IndVal) of bee species recorded in those areas (Dufrěne and Legendre, 1997). The IndVal is based on within-species abundance and occurrence comparisons in specified group. In addition, the IndVal method makes it possible to evaluate the strength of association between a given species and a given habitat. For this reason, IndVals were calculated for the whole collected material. If an IndVal was higher than 0.24, then the species was classified as strongly associated with the given habitat. The significance of IndVals was confirmed by a randomization procedure with 1000 replications. The above analyses were performed in the $R$ software environment ( $R$ Development Core Team, 2008) with the use of vegan (Oksanen et al., 2011) and labdsv libraries (Roberts, 2010).

To compare species diversity between the landscapes and between individual sites, we used species accumulation curves, which are analytical estimates (using the MaoTau estimator) of the expected number of species for the given number of samples (Gotelli and Colwell, 2001). As Gotelli and Colwell (2010) observed, lack of independence between samples can be neglected if rarefaction curves are functions of the number of samples. Comparison of species richness with unequal sampling effort, which incorporates sample-based rarefaction curves, is often used (Calvillo et al., 2010; Banaszak-Cibicka and Żmihorski, 2012; Żmihorski et al., 2013).

To estimate the true species number, we applied 2 estimators: Chao2 (Chao, 1984) and Jackknife2 (Burnham and Overton, 1979). Chao2 is a simple estimator of the true species number $\left(\mathrm{S}_{\text {chaor }}^{*}\right)$ in the community, based on the number of observed species $\left(\mathrm{S}_{\mathrm{obs}}\right)$ and rare species in the collected material, i.e., those present in a single sample (a) or in 2 samples (b):

$$
\mathrm{S}_{\text {chaor }}=\mathrm{S}_{\mathrm{obs}}+\left(\frac{\mathrm{a}^{2}}{2 \mathrm{~b}}\right)
$$

The estimator is the lower limit of the estimation and serves well for data sets where most information is concentrated in low-frequency classes, i.e., where a majority of species are rare. First- and second-order jackknife estimators are methods for reducing estimation error. Second-order jackknife estimates 


\section{Ј. APPC. SCL. VOL. 58 N NO. 12014}

the true species number on the basis of the number of species present in a single sample (uniques, $L$ ) and in 2 samples (duplicates, $M$ ):

$$
S_{\text {jack }}=S_{\text {obs }}+\left[\frac{L(2 n-3)}{n}-\frac{M(n-2)^{2}}{n(n-1)}\right]
$$

Individual-based rarefaction curves and expected species richness were calculated using Estimate $S$ software (Colwell, 2006).

To compare bee dominance structure between crop fields and semi-natural habitats, we used the evenness index $\left(E_{0}\right)$, which is not correlated with species number (S) (Smith and Wilson, 1996), as well as the slope angle of rank-abundance curves for most frequent species (top 40\%) in the community. The latter measure was used to describe the initial dominance structure. To obtain dominance structure, we used pooled samples for a given study site. Ranks $(x)$ of species were scaled $(x / S)$ so that the species with the highest contribution to the dominance structure had a value close to 0 while the rarest one had a value equal to $1 . E_{Q}$ was calculated according to the formula:

$E_{0}=-2 / \pi \arctan \left(b^{\prime}\right)$,

where $b^{\prime}$ is the slope of the inverse relationship between rank number and species abundance (species rank as a function of the logarithm of the number of individuals). $E_{0}$ values vary from 0 to 1 , where 1 denotes complete evenness of species in the community.

To obtain statistical differences between mean $E_{Q}$ values of fields and semi-natural habitats, we used simple Student's t tests, which test the null hypothesis of no group difference. The $F$ test was conducted to check equality of variances between compared groups.

\section{Study area}

Field research was conducted in 2 lowland regions separated by the Balkan Mountains (Stara Planina): the Danubian Plain and the Upper Thracian Plain. In 1986 - 1991, wild bees were studied in northern Bulgaria, in the central part of the Danubian Plain, about $15 \mathrm{~km}$ west of Pleven, mostly at several sites in the village of Krushovitsa. Both Pleven and Krushovitsa lie along the river Vit, a tributary of the Danube. The climate of the Pleven region is temperate continental, with a mean annual temperature of $11.5^{\circ} \mathrm{C}$. The coldest month is January (mean temperature $-2.4^{\circ} \mathrm{C}$ ), and the warmest is July (mean temperature $23.6^{\circ} \mathrm{C}$ ). Maximum temperature in summer is 42 $43^{\circ} \mathrm{C}$ whereas minimum in winter is $-24^{\circ} \mathrm{C}$. Westerly winds prevail in summer, and north-westerly in winter. In summer, dry days prevail (about 40 days).
Mean annual precipitation is $580-600 \mathrm{~mm}$, and monthly precipitation is highest in May - June. Snow cover lasts about 48 days. Autumn frost starts on October $17-21$, and late spring frost is recorded until April 6 - 10. In the northern part of the region, carbonate chernozems are most common, while in the south, typical chernozems and grey forest soils prevail. Alluvial soils are found in river valleys. Land relief is varied. Most of the area is covered by arable fields (79.3\%) and managed meadows (9.9\%), and the crop plants cultivated there include wheat, barley, maize, sugar beet, tobacco, sunflower, alfalfa, grape vines, and fruit trees.

In 1990 - 1992, field research was conducted in southern Bulgaria, in the Upper Thracian Plain, 10 - $12 \mathrm{~km}$ north of Plovdiv. The climate of the Plovdiv region is transitional continental with a mean annual temperature of about $12^{\circ} \mathrm{C}$. The coldest month is January (mean temperature $0.5^{\circ} \mathrm{C}$ ), and the warmest is July (mean temperature $22.7^{\circ} \mathrm{C}$ ). Maximum temperature in summer is $45^{\circ} \mathrm{C}$ while in winter the minimum is $-31.2^{\circ} \mathrm{C}$. Winds are mostly westerly or easterly, but about $50 \%$ of days are windless. Humidity is insufficient in summer, so it is a dry region and very dry in the south. Mean annual precipitation is $550-600 \mathrm{~mm}$, with the most abundant rainfall in May - June. Snow cover lasts only about 20 days, most often in January and February. The first autumn frost in the Upper Thracian Plain is usually recorded in late October or early November, and the late spring frost lasts until April. In the Plovdiv region, soils include alluvial (meadows), chernozems, and leached brown soils. Farmland accounts for $44.5 \%$ of the area, and the major crop plants are wheat, maize, tobacco, rice, peanut, grape vine, alfalfa, and fruit trees.

List of study sites:

\section{Danubian Plain, Pleven region (mostly Krusho- vitsa village)}

Field research was conducted at 9 sites. Some were marginal habitats: field roadside, ditch edge, covering small areas, usually belts 1 - $3 \mathrm{~m}$ wide, dominated by ruderal plants with some species characteristic of xerothermic grasslands and meadows. Larger areas were covered by verges of the Vit river valley, with mostly thermophilous vegetation, neighboring with fields. Fields of 4 selected crops also were investigated.

1. Valley and slope of the Vit river (Krushovitsa village). Samples $(64+11)$ collected April - October for 4 years (1986 - 1989).

2. Roadside. Samples (92) collected April - October for 4 years (1986 - 1989). 
3. Irrigation ditch near maize (Zea mays). Samples (53) collected April - September for 4 years (1986 - 1989).

4. Ditch edge. Samples (61) collected April - October for 5 years (1986 - 1990).

5. Alfalfa (Medicago sativa). Samples (51) collected from flowering seed production fields from June to early August for 5 years (1987 - 1991).

6. Sunflower (Helianthus annuus). Samples collected June - July for 2 years (1988 - 1989).

7. Oilseed rape (Brassica napus 'Elena', 'Tobin', and a Canadian cultivar). Samples (24) collected from April to mid-June for 2 years (1990 - 1991).

8. Radish (Raphanus sativus 'Omega'). Samples (11) collected from mid-May until mid-June for 2 years (1990 - 1991).

\section{Upper Thracian Plain, Plovdiv region}

As in the first region, the agricultural landscape was studied at 3 sites dominated by ruderal vegetation with some species typical of xerothermic grasslands. Trees and shrubs were very infrequent there.

1. Railway embankment, covered with grass and shrub communities. Samples (18) collected May September for 2 years (1990 - 1991).

2. Roadside. Samples (19) collected May - September for 2 years (1990 - 1991).

3. Ditch edge. Samples (22) collected May - September for 2 years (1990 - 1991).

\section{Others (near Sofia)}

Occasionally, bees were caught also at 2 other sites: 1. In the mountain massif of Vitosha near Sofia: edge of oak-hornbeam forest, roadside with rich herbaceous vegetation (Cichorium intybus, Vicia sp., Carduus nutans, Hypericum sp., Rubus sp.). Material collected in July 1986.

2. At the verges of Pancharevo, the southern suburbs of Sofia: pasture, meadow. Material collected in July 1986.

\section{RESULTS}

\section{Overall species diversity}

In total, 7046 wild bees of 232 species were collected and are now part of the personal collection of Prof. J. Banaszak in Bydgoszcz (Tab. 1). In the Pleven region, 206 species were found, whereas in the Plovdiv region, only 112 species were identified. Nonetheless, this disparity does not mean that the bee fauna of the Plovdiv region is poorer; material was collected there for only 2 years (total: 963 bees). In the Pleven region, it was collected for 6 years (total: 6007 bees). The expected cumulative number of wild bee species for these 2 regions indicates that species richness and diversity were similar in both. Thus, the smaller number of species reported from Plovdiv results from the shorter study period. Interpolation of results for the same number of samples suggests that species richness may be even greater for the agricultural landscape near Plovdiv. Species numbers estimated for Pleven and Plovdiv for similar numbers of samples do not differ significantly (overlapping 95\% confidence limits) (Fig. 1).

Good estimators of the true species number for a small number of samples are Jackknife 2 and Chao 2 (Collwell and Coddington, 1994). However, the applied estimators indicate much higher numbers of species for the landscape of Pleven (Chao2: 297.7, lower bound $95 \% \mathrm{Cl} 252.65$, upper bound 95\% Cl 383.62; Jackknife2: 311.39) than for Plovdiv (Chao2: 165.35, lower bound 95\% Cl 137.62, upper bound 95\% Cl 221.98; Jackknife2: 185.45). Based on the results of these estimators, species richness seems higher in the Pleven region.

\section{Contribution of Apis mellifera and wild bees}

Before discussing the dominance structure of wild bees, we should mention the contribution of honey bees to the studied ecosystems of the agricultural landscape. However, we discuss this question in detail in a separate publication (Banaszak et al., "unpubl. data"), for methodological reasons (it is difficult to match the number of wild bees observed during field research to species identified later), but also because the honey bee depends on location of apiaries and their size.

Honey bees were present at all the study sites, both in refuge habitats (e.g., roadsides, ditch edges, or river valleys) and in fields. However, the contribution of honey bees was highly variable, depending mostly on food resources. Thus, its abundance was variable in refuge habitats, where, e.g., large numbers of honey bees visited in spring the abundantly flowering Lepidium draba but in summer visited Knautia arvensis, Lamium purpureum, Echium vulgare, and others. The proportion of honey bees to wild bees varied between habitats and sampling sessions, as is well illustrated by Figure 2, where Apis mellifera was sometimes infrequent, whereas on another day and in a different place it accounted for $90 \%$ of the total. Its abundance varied from single individuals to 3 - 5 $\times 10^{3} / \mathrm{ha}$, respectively. As a rule, in crop fields, Apis mellifera was overwhelmingly dominant, accounting for $80 / 90-100 \%$ of the total catch, while wild bees contributed up to $20 \%$, as in the winter rape field. The density of Apis mellifera reached up to $50 \times 10^{3} /$ ha there compared to up to $1 \times 10^{3} /$ ha for wild bees (Fig. 2). 
Table 1.

List of species and number of individuals of Apiformes found at study sites

\begin{tabular}{|c|c|c|c|c|c|c|c|c|c|c|c|c|c|c|}
\hline \multirow[b]{2}{*}{ No. } & \multirow[b]{2}{*}{ Species } & \multicolumn{9}{|c|}{ Pleven region } & \multicolumn{3}{|c|}{ Plovdiv region } & \multirow[b]{2}{*}{ 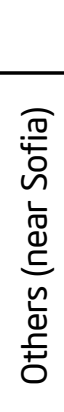 } \\
\hline & & 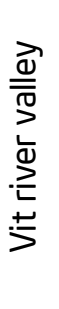 & $\begin{array}{l}\frac{0}{0} \\
\frac{0}{n} \\
\frac{\vec{d}}{\bar{N}}\end{array}$ & $\begin{array}{l}\frac{0}{n} \\
\frac{0}{0} \\
\frac{0}{\alpha}\end{array}$ & 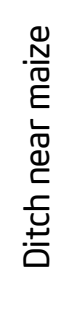 & 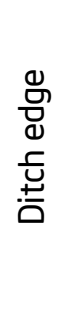 & 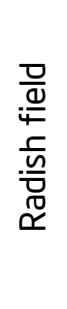 & 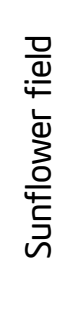 & 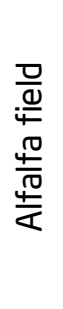 & 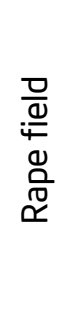 & 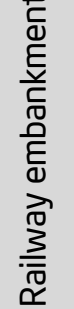 & 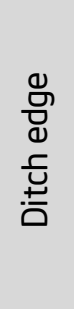 & 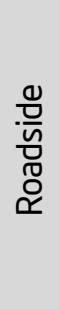 & \\
\hline & 1 & 2 & 3 & 4 & 5 & 6 & 7 & 8 & 9 & 10 & 11 & 12 & 13 & 14 \\
\hline 1 & $\begin{array}{l}\text { Colletes cunicularius (LINNEUS } \\
1761 \text { ) }\end{array}$ & & & 1 & & & & & & & & & & \\
\hline 2 & $\begin{array}{l}\text { Colletes daviesanus SMITH } \\
1846\end{array}$ & 1 & & & & & & & & & & & & \\
\hline 3 & $\begin{array}{l}\text { Colletes fodiens (GEOFFROY } \\
1765 \text { ) }\end{array}$ & & & & & & & & & & 1 & & & \\
\hline 4 & Colletes nasutus SMITH 1853 & 8 & & 1 & & & & & & & & & & \\
\hline 5 & $\begin{array}{l}\text { Colletes punctatus MOCSARY } \\
1877\end{array}$ & & & & & 19 & & & & & & & & \\
\hline 6 & Hylaeus annularis (KIRBY 1802) & 2 & & 1 & 1 & 2 & & & & & & & & \\
\hline 7 & $\begin{array}{l}\text { Hylaeus bisinuatus FÖRSTER } \\
1871\end{array}$ & & & 2 & 1 & & & & & & & 1 & & \\
\hline 8 & $\begin{array}{l}\text { Hylaeus communis NYLANDER } \\
1852\end{array}$ & & & 1 & & & & & & & & & & \\
\hline 9 & $\begin{array}{l}\text { Hylaeus confusus NYLANDER } \\
1852\end{array}$ & 1 & & 2 & & & & & & & & & & \\
\hline 10 & $\begin{array}{l}\text { Hylaeus gibbus SAUNDERS } \\
1850\end{array}$ & 2 & & & 2 & & & & & & & & & \\
\hline 11 & Hylaeus gredleri FÖRSTER 1871 & & & & & & & & & & & & 1 & \\
\hline 12 & Hylaeus kahri FÖRSTER 1871 & & & & & & & & & & 2 & & 1 & \\
\hline 13 & Hylaeus moricei (FRIESE 1898) & & 2 & 1 & & & & & & & & 1 & & \\
\hline 14 & $\begin{array}{l}\text { Hylaeus pictipes NYLANDER } \\
1852\end{array}$ & & & & & & 1 & & & & & & & \\
\hline 15 & $\begin{array}{l}\text { Hylaeus variegatus (FABRICIUS } \\
1798 \text { ) }\end{array}$ & 2 & & & & & & & & & 1 & 2 & 1 & \\
\hline 16 & $\begin{array}{l}\text { Andrena aeneiventris } \\
\text { MORAWITZ } 1872\end{array}$ & & & & & 5 & & & & 1 & & & & \\
\hline 17 & $\begin{array}{l}\text { Andrena alfkenella Perkins, } \\
1914\end{array}$ & & & & & & 2 & & & & & & & \\
\hline 18 & Andrena atrata FRIESE 1887 & & 2 & & 1 & & & 1 & 1 & & & & & \\
\hline 19 & $\begin{array}{l}\text { Andrena bicolor FABRICIUS } \\
1775\end{array}$ & & & & & & 1 & & & & & & & \\
\hline 20 & $\begin{array}{l}\text { Andrena bulgariensis WARNCKE } \\
1965\end{array}$ & & & & & & & & & & 1 & 4 & 3 & \\
\hline 21 & $\begin{array}{l}\text { Andrena carbonaria Linnaeus, } \\
1767\end{array}$ & & & & & & & & & & & & 1 & \\
\hline 22 & Andrena chrysopus PÉREZ 1903 & 1 & & & & & & & 1 & & & & & \\
\hline 23 & $\begin{array}{l}\text { Andrena chrysosceles (KIRBY } \\
\text { 1802) }\end{array}$ & & & & & & & & & & & & 1 & \\
\hline 24 & $\begin{array}{l}\text { Andrena cordialis MORAWITZ } \\
1878\end{array}$ & & & 8 & 6 & 2 & & & & 1 & & & & \\
\hline
\end{tabular}


Table 1 Continued.

List of species and number of individuals of Apiformes found at study sites

\begin{tabular}{|c|c|c|c|c|c|c|c|c|c|c|c|c|c|c|}
\hline & 1 & 2 & 3 & 4 & 5 & 6 & 7 & 8 & 9 & 10 & 11 & 12 & 13 & 14 \\
\hline 25 & $\begin{array}{l}\text { Andrena decipiens SCHENCK } \\
1859\end{array}$ & & 1 & & & 9 & & & 6 & & & & 3 & \\
\hline 26 & Andrena dorsata (KIRBY 1802) & 1 & & 6 & 1 & 3 & & & 1 & 1 & 1 & & 1 & \\
\hline 27 & $\begin{array}{l}\text { Andrena enslinella STOECKHERT } \\
1924\end{array}$ & & & & 1 & & & & & & & & & \\
\hline 28 & Andrena falsifica PERKINS 1915 & & & 2 & & 2 & & & & & & & & \\
\hline 29 & $\begin{array}{l}\text { Andrena figurata MORAWITZ } \\
1866\end{array}$ & & & & & & 1 & & & & & & & \\
\hline 30 & Andrena flavipes PANZER 1799 & 13 & 4 & 75 & 46 & 46 & 52 & 2 & 36 & 72 & 2 & 1 & 4 & 1 \\
\hline 31 & $\begin{array}{l}\text { Andrena floricola EVERSMANN } \\
1852\end{array}$ & & & & & & 2 & & & & 2 & & & \\
\hline 32 & $\begin{array}{l}\text { Andrena fulvata (STOECKHERT } \\
\text { 1930) }\end{array}$ & & & & & & & & 1 & & & & & \\
\hline 33 & $\begin{array}{l}\text { Andrena gelriae v. d. VECHT } \\
1927\end{array}$ & & & & & 4 & & & 3 & & & & & \\
\hline 34 & Andrena gravida IMHOFF 1832 & & & 1 & & & & & 1 & & & & & \\
\hline 35 & $\begin{array}{l}\text { Andrena haemorrhoa } \\
\text { (FABRICIUS 1781) }\end{array}$ & & & & & 1 & & & & & & & & 1 \\
\hline 36 & $\begin{array}{l}\text { Andrena hattorfiana (FABRICIUS } \\
\text { 1775) }\end{array}$ & 1 & 4 & & & & & & & & & & & \\
\hline 37 & $\begin{array}{l}\text { Andrena impunctata PÉREZ } \\
1895\end{array}$ & & & & & & & & & 1 & & & & \\
\hline 38 & Andrena jacobi Perkins, 1921 & & 1 & & & & & & & & & & & 1 \\
\hline 39 & Andrena labialis (KIRBY 1802) & & & & & 3 & & & & & & & & \\
\hline 40 & Andrena minutula (KIRBY 1802) & & & & 1 & & & & & 4 & & 1 & 2 & \\
\hline 41 & $\begin{array}{l}\text { Andrena minutuloides PERKINS } \\
1914\end{array}$ & 1 & & 5 & 3 & 2 & 1 & & & 3 & 2 & 11 & 3 & \\
\hline 42 & Andrena nana (KIRBY 1802) & & & & & & 1 & & & & & & & \\
\hline 43 & Andrena nasuta GIRAUD 1863 & 5 & & 1 & & & & & & & & & 1 & \\
\hline 44 & $\begin{array}{l}\text { Andrena nitidiuscula SCHENCK } \\
1853\end{array}$ & & & & 1 & & & & & & & & & \\
\hline 45 & Andrena niveata FRIESE 1887 & & & 1 & & 1 & & & & 1 & & & & \\
\hline 46 & Andrena obsoleta PÉREZ 1895 & & & 1 & 1 & 3 & 2 & & & 5 & & & & \\
\hline 47 & Andrena ovatula (KIRBY 1802) & 2 & & 8 & & 5 & 2 & & 17 & & & & 2 & \\
\hline 48 & Andrena pusilla PÉREZ 1903 & & & & & & 1 & & & 1 & & & & \\
\hline 49 & $\begin{array}{l}\text { Andrena saxonica STOECKHERT } \\
1935\end{array}$ & & & 1 & & & & & & & & & & \\
\hline 50 & $\begin{array}{l}\text { Andrena scita EVERSMANN } \\
1852\end{array}$ & & & & & & 1 & & & & & & & \\
\hline 51 & Andrena sericata IMHOFF 1868 & & & 1 & & 1 & & & & & & & & \\
\hline 52 & Andrena semilaevis PÉREZ 1903 & & & & & & 1 & & & & & & & \\
\hline 53 & $\begin{array}{l}\text { Andrena simontornyella } \\
\text { NOSKIEWICZ } 1939\end{array}$ & 1 & & & & & & & & & & & & \\
\hline 54 & Andrena sp. & & & & & & & & & 4 & & & & \\
\hline 55 & $\begin{array}{l}\text { Andrena spreta pusilla PÉREZ } \\
1902\end{array}$ & 1 & & & & & & & & & & & & \\
\hline 56 & $\begin{array}{l}\text { Andrena subopaca NYLANDER } \\
1848\end{array}$ & 2 & & 1 & & 1 & & & & & 7 & & 1 & \\
\hline 57 & Andrena tibialis (KIRBY 1802) & & & & & & & & & 1 & & & & \\
\hline 58 & Andrena tringa WARNCKE 1973 & & & & & 1 & 58 & & & 55 & & & 2 & \\
\hline
\end{tabular}


Table 1 Continued.

List of species and number of individuals of Apiformes found at study sites

\begin{tabular}{|c|c|c|c|c|c|c|c|c|c|c|c|c|c|c|}
\hline & 1 & 2 & 3 & 4 & 5 & 6 & 7 & 8 & 9 & 10 & 11 & 12 & 13 & 14 \\
\hline 59 & $\begin{array}{l}\text { Andrena truncatilabris } \\
\text { MORAWITZ } 1877\end{array}$ & 1 & & 14 & 9 & 17 & 41 & & 3 & 11 & & 1 & & \\
\hline 60 & $\begin{array}{l}\text { Andrena tsukubana HIRASHIMA } \\
1957\end{array}$ & & & 3 & 7 & 1 & & & & 2 & & & & \\
\hline 61 & Andrena variabilis SMITH 1853 & & & & & & & & 1 & & & & & \\
\hline 62 & $\begin{array}{l}\text { Andrena viridescens VIERECK } \\
1916\end{array}$ & 1 & & 1 & & & & & & & & & & \\
\hline 63 & Andrena wilkella (KIRBY 1802) & 4 & & & 5 & 3 & & 1 & 8 & 1 & & & & \\
\hline 64 & $\begin{array}{l}\text { Melitturga clavicornis (LATREILLE } \\
\text { 1806) }\end{array}$ & & & & & & & & 8 & & & & & \\
\hline 65 & $\begin{array}{l}\text { Panurginus labiatus (EVERSMANN } \\
\text { 1852) }\end{array}$ & 1 & & & & & & & & & & & & \\
\hline 66 & $\begin{array}{l}\text { Rhophitoides canus (EVERSMANN } \\
\text { 1852) }\end{array}$ & & & 2 & & 4 & & & 315 & & 1 & & & \\
\hline 67 & $\begin{array}{l}\text { Rophites hartmanni FRIESE } \\
1902\end{array}$ & 5 & & 1 & & & & & & & & 7 & 2 & \\
\hline 68 & $\begin{array}{l}\text { Rophites quinquespinosus } \\
\text { SPINOLA } 1808\end{array}$ & 2 & & 4 & & 1 & & & & & & & & \\
\hline 69 & $\begin{array}{l}\text { Systropha curvicornis (SCOPOLI } \\
1770 \text { ) }\end{array}$ & 3 & 3 & 6 & & 7 & & & 1 & & 59 & 36 & 26 & \\
\hline 70 & $\begin{array}{l}\text { Systropha planidens GIRAUD } \\
1861\end{array}$ & 1 & 10 & 7 & 11 & 72 & & & 6 & & 1 & & & 1 \\
\hline 71 & $\begin{array}{l}\text { Nomiapis diversipes (LATREILLE } \\
\text { 1806) }\end{array}$ & 3 & & 10 & 4 & 6 & & & 13 & & & & & \\
\hline 72 & $\begin{array}{l}\text { Nomioides minutissimus (ROSSI } \\
1790 \text { ) }\end{array}$ & & & 1 & & & & & & & & 1 & & \\
\hline 73 & $\begin{array}{l}\text { Halictus cochlearitarsis DOURS } \\
1872\end{array}$ & 3 & 1 & 3 & & & & & & & 1 & & 2 & \\
\hline 74 & $\begin{array}{l}\text { Halictus compressus } \\
\text { WALCKENAER } 1802\end{array}$ & 2 & & 2 & & 2 & & & & & & & & \\
\hline 75 & Halictus maculatus SMITH 1848 & 11 & 1 & 99 & 122 & 91 & & 1 & 7 & 7 & 10 & 7 & 15 & 1 \\
\hline 76 & $\begin{array}{l}\text { Halictus patellatus MORAWITZ } \\
1873\end{array}$ & & & & 1 & & & & 2 & 1 & 1 & & & \\
\hline 77 & $\begin{array}{l}\text { Halictus pollinosus cariniventris } \\
\text { MORAWITZ } 1876\end{array}$ & 1 & & & & & & & & & & & & \\
\hline 78 & $\begin{array}{l}\text { Halictus ponticus BLÜTHGEN } \\
1934\end{array}$ & & & 2 & & 2 & & & & & & & & \\
\hline 79 & $\begin{array}{l}\text { Halictus quadricinctus (FABRICIUS } \\
1776 \text { ) }\end{array}$ & 38 & 7 & 67 & 5 & 19 & & 42 & & 1 & & & & \\
\hline 80 & Halictus resurgens NURSE 1903 & 58 & & 38 & 6 & 17 & 1 & 7 & 21 & 1 & 2 & 68 & 45 & \\
\hline 81 & Halictus scabiosae (ROSSI 1790) & 9 & 2 & 14 & 2 & 2 & & 5 & & & & & 3 & \\
\hline 82 & $\begin{array}{l}\text { Halictus sexcinctus (FABRICIUS, } \\
1775 \text { ) }\end{array}$ & 23 & 3 & 9 & 1 & & & 1 & & & & & 5 & \\
\hline 83 & Halictus simplex/compressus & 29 & 7 & 127 & 91 & 133 & 3 & 2 & 133 & 7 & 6 & 4 & 16 & \\
\hline 84 & $\begin{array}{l}\text { Halictus tectus RADOSZKOWSKI } \\
1875\end{array}$ & & & & & & & & & & & & 1 & \\
\hline 85 & $\begin{array}{l}\text { Seladonia kessleri (BRAMSON } \\
\text { 1879) }\end{array}$ & & 8 & 9 & 2 & 12 & 1 & & & 3 & & & & \\
\hline 86 & $\begin{array}{l}\text { Seladonia pollinosa (SICHEL } \\
\text { 1860) }\end{array}$ & 1 & & & & 1 & & 1 & & 1 & 1 & & 3 & \\
\hline
\end{tabular}


Table 1 Continued.

List of species and number of individuals of Apiformes found at study sites

\begin{tabular}{|c|c|c|c|c|c|c|c|c|c|c|c|c|c|c|}
\hline & 1 & 2 & 3 & 4 & 5 & 6 & 7 & 8 & 9 & 10 & 11 & 12 & 13 & 14 \\
\hline 87 & $\begin{array}{l}\text { Seladonia semitecta (MORAWITZ } \\
\text { 1874) }\end{array}$ & & & & & 1 & & & 1 & & & & & \\
\hline 88 & $\begin{array}{l}\text { Seladonia smaragdula (VACHAL } \\
\text { 1895) }\end{array}$ & & 1 & & 8 & 2 & & & 5 & & & & 1 & \\
\hline 89 & $\begin{array}{l}\text { Seladonia subaurata (ROSSI } \\
\text { 1792) }\end{array}$ & 6 & & 10 & 17 & 5 & & & 3 & 1 & & & & \\
\hline 90 & $\begin{array}{l}\text { Seladonia tataricus (BLÜTHGEN } \\
\text { 1933) }\end{array}$ & & & 3 & & & & & & & & & & \\
\hline 91 & $\begin{array}{l}\text { Seladonia tumulorum (LINNAEUS } \\
1758 \text { ) }\end{array}$ & 1 & & 2 & 1 & 1 & & & & & & & & \\
\hline 92 & $\begin{array}{l}\text { Lasioglossum aegyptiellum } \\
\text { (STRAND 1909) }\end{array}$ & & & 1 & & & & & & & & & & \\
\hline 93 & $\begin{array}{l}\text { Lasioglossum cristula donatum } \\
\text { (WARNCKE 1975) }\end{array}$ & 1 & & & 1 & & & & & & 5 & 7 & 4 & \\
\hline 94 & $\begin{array}{l}\text { Lasioglossum discum (SMITH } \\
\text { 1853) }\end{array}$ & 3 & & 2 & & & & & & & & & 2 & \\
\hline 95 & $\begin{array}{l}\text { Lasioglossum lativentre } \\
\text { (SCHENCK 1853) }\end{array}$ & & & & 1 & & & & & & & & & \\
\hline 96 & $\begin{array}{l}\text { Lasioglossum leucozonium } \\
\text { (SCHRANK 1781) }\end{array}$ & 6 & 6 & 15 & 22 & 13 & 4 & & & 1 & 3 & & & \\
\hline 97 & $\begin{array}{l}\text { Lasioglossum morbillosum } \\
\text { (KRIECHBAUMER 1873) }\end{array}$ & 18 & & 38 & 14 & 20 & 2 & 1 & 1 & 2 & 3 & 7 & 10 & \\
\hline 98 & $\begin{array}{l}\text { Lasioglossum nitidum (PANZER } \\
\text { 1798) }\end{array}$ & & & 1 & & & & & & & & & & \\
\hline 99 & $\begin{array}{l}\text { Lasioglossum pseudomorbillo- } \\
\text { sum (EBMER 1970) }\end{array}$ & 13 & 3 & 29 & 2 & 21 & 1 & & 3 & 3 & & 1 & 3 & \\
\hline 100 & $\begin{array}{l}\text { Lasioglossum aegyptiellum } \\
\text { (STRAND 1909) }\end{array}$ & & & 1 & & & & & & & & & & \\
\hline 101 & $\begin{array}{l}\text { Lasioglossum quadrinotatum } \\
\text { (KIRBY 1802) }\end{array}$ & & & 3 & 1 & & & & & & & & & \\
\hline 102 & $\begin{array}{l}\text { Lasioglossum sexnotatum } \\
\text { (KIRBY 1802) }\end{array}$ & & & 2 & & & 2 & & & & & & & \\
\hline 103 & $\begin{array}{l}\text { Lasioglossum xanthopus (KIRBY } \\
\text { 1802) }\end{array}$ & & 1 & & & & & & & & & & & \\
\hline 104 & $\begin{array}{l}\text { Lasioglossum zonulum (SMITH } \\
\text { 1848) }\end{array}$ & & & & 6 & 1 & & & & & & & & \\
\hline 105 & $\begin{array}{l}\text { Evylaeus albipes (FABRICIUS } \\
\text { 1781) }\end{array}$ & 2 & & & & 2 & & & & & & & & 3 \\
\hline 106 & $\begin{array}{l}\text { Evylaeus calceatus (SCOPOLI } \\
1763 \text { ) }\end{array}$ & 9 & 2 & 14 & 6 & 3 & & & & 2 & & & & 2 \\
\hline 107 & $\begin{array}{l}\text { Evylaeus clypearis (SCHENCK } \\
\text { 1853) }\end{array}$ & 1 & & 2 & & 3 & & & & & 3 & & 4 & \\
\hline 108 & $\begin{array}{l}\text { Evylaeus corvinum (MORAWITZ } \\
\text { 1878) }\end{array}$ & 18 & 33 & 70 & 32 & 107 & 4 & 1 & & 30 & 2 & 1 & & \\
\hline 109 & $\begin{array}{l}\text { Evylaeus crassepunctatum } \\
\text { (BLÜTHGEN 1923) }\end{array}$ & & & 2 & 1 & 2 & & & & 2 & & 1 & & \\
\hline 110 & $\begin{array}{l}\text { Evylaeus fulvicornis (KIRBY } \\
\text { 1802) }\end{array}$ & 1 & 1 & & 3 & 2 & & & & & & & & \\
\hline 111 & $\begin{array}{l}\text { Evylaeus glabriusculus } \\
\text { (MORAWITZ 1872) }\end{array}$ & & 4 & 14 & 61 & 5 & 14 & & 11 & 51 & & & & \\
\hline
\end{tabular}




\section{J. APIC. SCLI. VOL. 58 N NO. 12014}

Table 1 Continued.

List of species and number of individuals of Apiformes found at study sites

\begin{tabular}{|c|c|c|c|c|c|c|c|c|c|c|c|c|c|c|}
\hline & 1 & 2 & 3 & 4 & 5 & 6 & 7 & 8 & 9 & 10 & 11 & 12 & 13 & 14 \\
\hline 112 & $\begin{array}{l}\text { Evylaeus griseolum (MORAWITZ } \\
\text { 1872) }\end{array}$ & 4 & 4 & & 1 & 1 & & & 1 & 3 & 1 & 2 & 4 & \\
\hline 113 & $\begin{array}{l}\text { Evylaeus interruptus (PANZER } \\
1798 \text { ) }\end{array}$ & & & & & & & & & & 6 & 6 & 1 & \\
\hline 114 & $\begin{array}{l}\text { Evylaeus laticeps (SCHENCK } \\
\text { 1868) }\end{array}$ & 3 & 2 & 1 & & & 2 & & & 3 & & & & \\
\hline 115 & Evylaeus leucopus (KIRBY 1802) & & & & & & & & & & & 3 & & \\
\hline 116 & $\begin{array}{l}\text { Evylaeus lineare (SCHENCK } \\
\text { 1868) }\end{array}$ & 13 & & 7 & 5 & 10 & 1 & 2 & 1 & 1 & 3 & 3 & 1 & \\
\hline 117 & $\begin{array}{l}\text { Evylaeus linearis (SCHENCK } \\
\text { 1868) }\end{array}$ & 3 & 1 & 3 & & 5 & & & & & & 1 & & \\
\hline 118 & $\begin{array}{l}\text { Evylaeus lucidulus (SCHENCK } \\
\text { 1861) }\end{array}$ & & & 3 & 19 & & 1 & & & 12 & 22 & 27 & 16 & \\
\hline 119 & $\begin{array}{l}\text { Evylaeus malachurus (KIRBY } \\
\text { 1802) }\end{array}$ & 143 & 34 & 166 & 111 & 85 & 26 & 60 & 5 & 58 & 10 & 2 & 1 & 1 \\
\hline 120 & $\begin{array}{l}\text { Evylaeus marginatus (BRULLÉ } \\
\text { 1832) }\end{array}$ & 1 & 1 & 1 & 1 & & & & & 7 & & & & \\
\hline 121 & $\begin{array}{l}\text { Evylaeus morio (FABRICIUS } \\
1793)\end{array}$ & 1 & & & & & & & & & & & & \\
\hline 122 & $\begin{array}{l}\text { Evylaeus nigripes (LEPELETIER } \\
\text { 1841) }\end{array}$ & 2 & & 8 & & & & & & & 1 & & 1 & \\
\hline 123 & $\begin{array}{l}\text { Evylaeus pauxillus (SCHENCK } \\
\text { 1853) }\end{array}$ & 4 & 2 & 31 & 46 & 21 & 4 & 1 & 5 & 44 & & & 1 & \\
\hline 124 & $\begin{array}{l}\text { Evylaeus politus (SCHENCK } \\
\text { 1853) }\end{array}$ & 1 & & 1 & & & & & & 2 & 8 & 11 & 1 & \\
\hline 125 & $\begin{array}{l}\text { Evylaeus pressi thorax (EBMER } \\
\text { 1974) }\end{array}$ & & & & & & 1 & & & & & & & \\
\hline 126 & $\begin{array}{l}\text { Evylaeus puncticolle (MORAWITZ } \\
\text { 1872) }\end{array}$ & & & & & & & & & & & & 1 & \\
\hline 127 & $\begin{array}{l}\text { Evylaeus sexstriqatus (SCHENCK } \\
1868 \text { ) }\end{array}$ & 1 & & & & & & & & & & & & \\
\hline 128 & $\begin{array}{l}\text { Evylaeus truncaticolle } \\
\text { (MORAWITZ 1878) }\end{array}$ & 16 & 12 & 23 & 10 & 12 & & 2 & & & 2 & 21 & 62 & \\
\hline 129 & $\begin{array}{l}\text { Evylaeus truncaticollis } \\
\text { (MORAWITZ 1877) }\end{array}$ & & 1 & & & & & & & & & 2 & 1 & \\
\hline 130 & $\begin{array}{l}\text { Evylaeus villosulus (KIRBY } \\
1802 \text { ) }\end{array}$ & 2 & 1 & 4 & 1 & 2 & 3 & & & & 1 & & 1 & \\
\hline 131 & $\begin{array}{l}\text { Sphecodes crassus THOMSON } \\
1870\end{array}$ & & & & & & & & & 1 & & & & \\
\hline 132 & $\begin{array}{l}\text { Sphecodes gibbus (LINNAEUS } \\
1758)\end{array}$ & & & 2 & 5 & & & & & & & 1 & & \\
\hline 133 & $\begin{array}{l}\text { Sphecodes longulus HAGENS } \\
1882\end{array}$ & & & & & & & & & & & 3 & & \\
\hline 134 & $\begin{array}{l}\text { Sphecodes monilicornis (KIRBY } \\
1802 \text { ) }\end{array}$ & & & & & & & & & & & 2 & & \\
\hline 135 & $\begin{array}{l}\text { Sphecodes pellucidus SMITH } \\
1845\end{array}$ & & & & & & & & & & & 1 & & \\
\hline 136 & $\begin{array}{l}\text { Sphecodes rufiventris (PANZER } \\
1798 \text { ) }\end{array}$ & & & & & & & & & & & 1 & & \\
\hline 137 & Sphecodes sp. & 1 & & & & & & & & & & & & \\
\hline 138 & Melitta leporina (PANZER 1799) & 2 & 2 & 2 & 1 & 7 & & & 134 & & & & & \\
\hline
\end{tabular}


List of species and number of individuals of Apiformes found at study sites

Table 1 Continued.

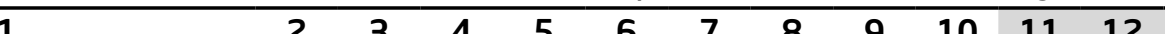

\begin{tabular}{|c|c|c|c|c|c|c|c|c|c|c|c|c|c|}
\hline & 1 & 2 & 3 & 4 & 5 & 6 & 7 & 8 & $9 \quad 10$ & 11 & 12 & 13 & $\overline{14}$ \\
\hline 139 & Melitta nigricans ALFKEN 1905 & & 21 & & & & & & & & & & \\
\hline 140 & Melitta tricincta KIRBY 1802 & 1 & & & & & & & & & & & \\
\hline 141 & $\begin{array}{l}\text { Macropis fulvipes (FABRICIUS } \\
\text { 1804) }\end{array}$ & & & & & & & & & & & & 2 \\
\hline 142 & $\begin{array}{l}\text { Dasypoda hirtipes (HARRIS } \\
\text { 1780) }\end{array}$ & 29 & 14 & 27 & 2 & & & & 1 & 2 & 15 & & \\
\hline 143 & $\begin{array}{l}\text { Lithurgus chrysurus FONSCO- } \\
\text { LOMBE } 1834\end{array}$ & 3 & & 3 & & & & & & & 7 & 2 & \\
\hline 144 & $\begin{array}{l}\text { Paranthidiellum lituratum } \\
\text { (PANZER 1801) }\end{array}$ & 6 & 3 & 3 & 1 & 5 & & & & & 5 & 9 & \\
\hline 145 & $\begin{array}{l}\text { Paraanthidiellum tenellum } \\
\text { (MOCSÁRY 1881) }\end{array}$ & 1 & 1 & 5 & & 1 & & & & 1 & 2 & 2 & \\
\hline 146 & $\begin{array}{l}\text { Anthidium florentinum } \\
\text { (FABRICIUS 1775) }\end{array}$ & & & & & & & & 1 & 2 & 6 & & \\
\hline 147 & $\begin{array}{l}\text { Anthidium manicatum } \\
\text { (LINNAEUS 1758) }\end{array}$ & 1 & & 6 & & & & & & 1 & 2 & & \\
\hline 148 & $\begin{array}{l}\text { Anthidium punctatum LATREILLE } \\
1809\end{array}$ & 1 & & & & & & & & & & & \\
\hline 149 & $\begin{array}{l}\text { Anthidiellum strigatum (PANZER } \\
\text { 1805) }\end{array}$ & 1 & & & & & & & & & & 1 & \\
\hline 150 & $\begin{array}{l}\text { Heriades crenulatus NYLANDER } \\
1856\end{array}$ & & & & & & & & & & 26 & 15 & \\
\hline 151 & $\begin{array}{l}\text { Chelostoma grande (NYLANDER } \\
\text { 1852) }\end{array}$ & 1 & & & & & & & & 1 & & & \\
\hline 152 & $\begin{array}{l}\text { Anthocopa andrenoides } \\
\text { (SPINOLA 1808) }\end{array}$ & & & & & & & & & 1 & & & \\
\hline 153 & $\begin{array}{l}\text { Anthocopa bidentata } \\
\text { (MORAWITZ 1876) }\end{array}$ & & & 2 & & & & & & & 6 & 3 & \\
\hline 154 & $\begin{array}{l}\text { Anthocopa spinulosa (KIRBY } \\
\text { 1802) }\end{array}$ & 1 & & 1 & & & & & & 1 & 2 & 1 & \\
\hline 155 & Hoplitis adunca (PANZER 1798) & & 1 & & & & & & & 1 & & & \\
\hline 156 & $\begin{array}{l}\text { Hoplitis leucomelana (KIRBY } \\
\text { 1802) }\end{array}$ & & & 1 & & & & & & & & 1 & \\
\hline 157 & $\begin{array}{l}\text { Hoplitis manicata (MORICE } \\
\text { 1901) }\end{array}$ & & & & & & & & & & & 1 & \\
\hline 158 & $\begin{array}{l}\text { Hoplitis rufohirta LATREILLE } \\
1811\end{array}$ & 1 & & 17 & 1 & 3 & & & 1 & & & 4 & \\
\hline 159 & $\begin{array}{l}\text { Hoplitis tridentata (DUFOUR and } \\
\text { PERRIS 1840) }\end{array}$ & & & & & & & & & & & 3 & \\
\hline 160 & Osmia aurulenta (PANZER 1799) & 11 & & 14 & & 5 & & & 6 & 2 & 1 & 7 & \\
\hline 161 & $\begin{array}{l}\text { Osmia brevicornis (FABRICIUS } \\
1798 \text { ) }\end{array}$ & 2 & & & & & & & & & & & \\
\hline 162 & $\begin{array}{l}\text { Osmia caerulescens (LINNAEUS } \\
1758 \text { ) }\end{array}$ & 5 & 1 & 3 & & & 1 & & 4 & 6 & 5 & & \\
\hline 163 & $\begin{array}{l}\text { Osmia cornuta (LATREILLE } \\
\text { 1805) }\end{array}$ & & & & & & & & & & & & 6 \\
\hline 164 & $\begin{array}{l}\text { Osmia fulviventris (PANZER } \\
\text { 1798) }\end{array}$ & & & & & & & & & 1 & & 1 & \\
\hline 165 & $\begin{array}{l}\text { Megachile apicalis SPINOLA } \\
1808\end{array}$ & & & 2 & & 2 & & & & & 4 & 1 & \\
\hline 166 & $\begin{array}{l}\text { Megachile centuncularis } \\
\text { (LINNAEUS 1758) }\end{array}$ & 7 & 4 & 1 & 3 & & & & & & 1 & & \\
\hline
\end{tabular}

in 
Table 1 Continued.

List of species and number of individuals of Apiformes found at study sites

\begin{tabular}{|c|c|c|c|c|c|c|c|c|c|c|c|c|c|c|}
\hline & 1 & 2 & 3 & 4 & 5 & 6 & 7 & 8 & 9 & 10 & 11 & 12 & 13 & 14 \\
\hline 167 & $\begin{array}{l}\text { Megachile deceptoria PÉREZ } \\
1890\end{array}$ & & 1 & 1 & & 1 & & & 1 & & & & 1 & \\
\hline 168 & $\begin{array}{l}\text { Megachile leucomalla } \\
\text { GERSTÄCKER } 1869\end{array}$ & & & & & & & & 1 & & & & & \\
\hline 169 & Megachile pilidens ALFKEN 1923 & 21 & 7 & 25 & 6 & 20 & & & 4 & & 6 & 2 & 3 & \\
\hline 170 & $\begin{array}{l}\text { Megachile rotundata (FABRICIUS } \\
\text { 1784) }\end{array}$ & & & & & & & & 5 & & & & & \\
\hline 171 & $\begin{array}{l}\text { Megachile versicolor SMITH } \\
1844\end{array}$ & 5 & & 4 & & 1 & & & & & & & & \\
\hline 172 & $\begin{array}{l}\text { Coelioxys afra LEPELETIER } \\
1841\end{array}$ & 1 & & 1 & & & & & 2 & & 1 & & 1 & \\
\hline 173 & $\begin{array}{l}\text { Paradioxys pannonica MOCSÁRY } \\
1877\end{array}$ & & & 2 & & & & & & & & & & \\
\hline 174 & $\begin{array}{l}\text { Anthophora crinipes SMITH } \\
1854\end{array}$ & & & & & & & & & & 16 & & & \\
\hline 175 & $\begin{array}{l}\text { Anthophora plumipes (PALLAS } \\
\text { 1772) }\end{array}$ & & & & & & & & & 1 & 2 & & & \\
\hline 176 & $\begin{array}{l}\text { Amegilla magnilabris (FEDT- } \\
\text { SCHENKO 1875) }\end{array}$ & 3 & & 1 & & & & & & & & & & \\
\hline 177 & $\begin{array}{l}\text { Amegilla quadrifasciata (VILLERS } \\
\text { 1789) }\end{array}$ & 1 & 2 & & & & & & & & & & & \\
\hline 178 & $\begin{array}{l}\text { Amegilla salviae (MORAWITZ } \\
\text { 1876) }\end{array}$ & & & & & 1 & & & & & & & & \\
\hline 179 & Eucera chrysopyga PéREZ 1879 & 23 & & 52 & 10 & 17 & 1 & & 30 & 24 & 1 & & & \\
\hline 180 & $\begin{array}{l}\text { Eucera clypeata ERICHSON } \\
1835\end{array}$ & 3 & & 2 & 1 & 4 & 1 & 1 & 107 & 3 & & & & \\
\hline 181 & $\begin{array}{l}\text { Eucera dalmatica LEPELETIER } \\
1841\end{array}$ & & & 1 & & & & & & & & & & \\
\hline 182 & $\begin{array}{l}\text { Eucera longicornis (LINNAEUS } \\
1758 \text { ) }\end{array}$ & 7 & 3 & 13 & 7 & 3 & & & 2 & & & & & \\
\hline 183 & $\begin{array}{l}\text { Eucera nigrilabris LEPELETIER } \\
1841\end{array}$ & & & & 2 & & & & & & & & & \\
\hline 184 & $\begin{array}{l}\text { Eucera nitidiventris MOCSÁRY } \\
1879\end{array}$ & 4 & & 28 & 7 & 5 & 1 & & 32 & 4 & 1 & & & 1 \\
\hline 185 & $\begin{array}{l}\text { Eucera parvicornis MOCSÁRY } \\
1878\end{array}$ & & & 1 & & & & & & & & & & \\
\hline 186 & Eucera seminuda BRULLË1832 & & & & 1 & 1 & & & & & & & & 1 \\
\hline 187 & Eucera taurica MORAWITZ 1871 & & 1 & 11 & & & & & & & & & & \\
\hline 188 & $\begin{array}{l}\text { Eucera tuberculata (FABRICIUS } \\
1793 \text { ) }\end{array}$ & 3 & & 8 & 7 & 2 & & & 5 & 2 & & & & \\
\hline 189 & $\begin{array}{l}\text { Tetralonia alternans } \\
\text { BRULLË1832 }\end{array}$ & & & & & & & & & & & 1 & & \\
\hline 190 & Tetralonia dentata (KLUG 1835) & 1 & & & & & & & & & & & 1 & \\
\hline 191 & $\begin{array}{l}\text { Tetralonia macroglossa ILLIGER } \\
1806\end{array}$ & 9 & 5 & 1 & & & & & & & 1 & & 2 & \\
\hline 192 & $\begin{array}{l}\text { Tetralonia nana MORAWITZ } \\
1874\end{array}$ & 20 & 4 & 1 & & 1 & & & & & & & & \\
\hline 193 & $\begin{array}{l}\text { Tetralonia pollinosa (LEPELETIER } \\
\text { 1841) }\end{array}$ & 7 & & 7 & 1 & & & & & & & & & \\
\hline 194 & $\begin{array}{l}\text { Tetralonia ruficornis (FABRICIUS } \\
\text { 1804) }\end{array}$ & 2 & & 1 & & & & & & & & 1 & 3 & \\
\hline
\end{tabular}


List of species and number of individuals of Apiformes found at study sites

\begin{tabular}{|c|c|c|c|c|c|c|c|c|c|c|c|c|c|c|}
\hline & 1 & 2 & 3 & 4 & 5 & 6 & 7 & 8 & 9 & 10 & 11 & 12 & 13 & 14 \\
\hline 195 & $\begin{array}{l}\text { Tetralonia salicariae } \\
\text { (LEPELETIER 1841) }\end{array}$ & 5 & 3 & & & & & & & & & & & \\
\hline 196 & $\begin{array}{l}\text { Tetralonia scabiosae (MOCSÁRY } \\
\text { 1881) }\end{array}$ & & 1 & & & 7 & & & & & 3 & & & \\
\hline 197 & Tetralonia sp. & 3 & & & & 1 & & & & & & & & \\
\hline 198 & $\begin{array}{l}\text { Xylocopa valga GERSTAECKER, } \\
1872\end{array}$ & & & & & & & & & 1 & 1 & & & \\
\hline 199 & Ceratina acuta FRIESE 1896 & & & & & 1 & & & & & & & & \\
\hline 200 & $\begin{array}{l}\text { Ceratina callosa (FABRICIUS } \\
1794)\end{array}$ & 7 & 3 & 1 & 1 & & & & & & 1 & & 1 & \\
\hline 201 & $\begin{array}{l}\text { Ceratina chalcites GERMAR } \\
1839\end{array}$ & & 5 & & & & & & & & 1 & 1 & 2 & \\
\hline 202 & $\begin{array}{l}\text { Ceratina cucurbitina (ROSSI } \\
1792 \text { ) }\end{array}$ & & 1 & & & & & & & & 15 & 1 & 3 & \\
\hline 203 & Ceratina cyanea (KIRBY 1802) & 29 & 1 & 12 & 8 & 7 & 1 & & & 2 & 4 & 6 & 2 & \\
\hline 204 & $\begin{array}{l}\text { Ceratina dallotorreana FRIESE, } \\
1896\end{array}$ & 2 & & 1 & 3 & 2 & & & & & 3 & 1 & & \\
\hline 205 & Ceratina parvula SMITH 1854 & & & & & & & & & & 1 & 2 & 1 & \\
\hline 206 & $\begin{array}{l}\text { Nomada basalis HERRICH- } \\
\text { SCHÄFFER } 1839\end{array}$ & & & & & & 4 & & 1 & 1 & & & & \\
\hline 207 & $\begin{array}{l}\text { Nomada emarginata MORAWITZ } \\
1877\end{array}$ & & 3 & & & & & & & & & & & \\
\hline 208 & $\begin{array}{l}\text { Nomada fabriciana (LINNAEUS } \\
\text { 1767) }\end{array}$ & & & & 1 & & & & & & & & & \\
\hline 209 & Nomada flavopicta (KIRBY 1802) & 1 & & & & & & & & & & & & \\
\hline 210 & Nomada fucata PANZER 1798 & 2 & & 1 & 2 & 1 & & & & & 1 & & & \\
\hline 211 & $\begin{array}{l}\text { Nomada kohli SCHMIEDEKNECHT } \\
1882\end{array}$ & 1 & & 1 & 1 & 1 & & & & 1 & & 1 & & \\
\hline 212 & $\begin{array}{l}\text { Nomada lathburiana (KIRBY } \\
\text { 1802) }\end{array}$ & 1 & & & & & & & & & & & & \\
\hline 213 & $\begin{array}{l}\text { Nomada nobilis HERRICH- } \\
\text { SCHÄFFER } 1839\end{array}$ & & & & & & 7 & & 1 & 9 & & & & \\
\hline 214 & $\begin{array}{l}\text { Nomada pleurosticta HERRICH- } \\
\text { SCHÄFFER } 1839\end{array}$ & & & 1 & & & & & 1 & & & & & \\
\hline & $\begin{array}{l}\text { Nomada sexfasciata PANZER } \\
1799\end{array}$ & & & & & & & & 1 & & & & & \\
\hline & Nomada stigma FABRICIUS 1804 & & & & & & & & 1 & & & & & \\
\hline 217 & $\begin{array}{l}\text { Biastes brevicornis (PANZER } \\
1798 \text { ) }\end{array}$ & & & & & 3 & & & & & & 1 & & \\
\hline 218 & $\begin{array}{l}\text { Bombus argillaceus (SCOPOLI } \\
1763 \text { ) }\end{array}$ & 1 & & 4 & 1 & & & & & & & & & 2 \\
\hline 219 & $\begin{array}{l}\text { Bombus cryptarum (FABRICIUS } \\
1775 \text { ) }\end{array}$ & & & & 1 & & & 2 & & & 1 & 1 & & \\
\hline 220 & $\begin{array}{l}\text { Bombus hortorum (LINNAEUS } \\
1761 \text { ) }\end{array}$ & 14 & & 74 & & 6 & & 5 & & & 1 & 1 & & 8 \\
\hline 221 & Bombus humilis ILLIGER 1806 & 1 & & & & & & & & & & & & \\
\hline 222 & $\begin{array}{l}\text { Bombus hypnorum (LINNAEUS } \\
1758 \text { ) }\end{array}$ & & & & & & & & & & & & & 2 \\
\hline 223 & $\begin{array}{l}\text { Bombus lapidarius (LINNAEUS } \\
\text { 1758) }\end{array}$ & & & & & & & & & & & & & 6 \\
\hline
\end{tabular}


Table 1 Continued.

List of species and number of individuals of Apiformes found at study sites

\begin{tabular}{|c|c|c|c|c|c|c|c|c|c|c|c|c|c|}
\hline 1 & 2 & 3 & 4 & 5 & 6 & 7 & 8 & 9 & 10 & 11 & 12 & 13 & 14 \\
\hline $\begin{array}{ll}224 & \begin{array}{l}\text { Bombus lucorum (LINNAEUS } \\
1761)\end{array}\end{array}$ & 3 & & & 1 & & & & & & & 3 & & 4 \\
\hline $225 \begin{array}{l}\text { Bombus muscorum (LINNAEUS } \\
1758)\end{array}$ & & & & & & & & & & & & & 1 \\
\hline $226 \begin{array}{l}\text { Bombus pascuorum (SCOPOLI } \\
1763)\end{array}$ & 1 & & 1 & & & & & & & & & & 17 \\
\hline $227 \begin{array}{l}\text { Bombus pratorum (LINNAEUS } \\
1761 \text { ) }\end{array}$ & 1 & & 1 & & & & & & 1 & & & & 1 \\
\hline $\begin{array}{ll}228 & \begin{array}{l}\text { Bombus ruderarius (MÜLLER } \\
1776)\end{array} \\
\end{array}$ & 2 & & 2 & & & & 1 & & & & & & \\
\hline $229 \begin{array}{l}\text { Bombus subterraneus } \\
\text { (LINNAEUS 1758) }\end{array}$ & & & & & & & & & & & & & 1 \\
\hline $230 \begin{array}{l}\text { Bombus sylvarum (LINNAEUS } \\
1761 \text { ) }\end{array}$ & 17 & & 28 & 1 & 7 & & 1 & 10 & & & & & \\
\hline $\begin{array}{ll}231 & \begin{array}{l}\text { Bombus terrestris (LINNAEUS } \\
1758)\end{array}\end{array}$ & 5 & & 18 & 3 & 5 & & 23 & 2 & & 19 & 7 & 4 & 13 \\
\hline $\begin{array}{ll}232 & \begin{array}{l}\text { Bombus veteranus (FABRICIUS } \\
1793)\end{array}\end{array}$ & 2 & & & & & & & & & & & & \\
\hline 233 Apis mellifera LINNAEUS, 1758 & + & + & + & + & + & + & + & + & + & + & + & + & + \\
\hline $\begin{array}{l}\text { Number of individuals in different } \\
\text { habitats }\end{array}$ & 795 & 252 & 1390 & 779 & 944 & 253 & 163 & 974 & 457 & 269 & 359 & 335 & 76 \\
\hline Total number of individuals & \multicolumn{13}{|c|}{7046} \\
\hline $\begin{array}{c}\text { Number of species in different } \\
\text { habitats }\end{array}$ & 112 & 55 & 115 & 75 & 86 & 39 & 23 & 55 & 52 & 63 & 62 & 70 & 23 \\
\hline $\begin{array}{c}\text { Number of species in different } \\
\text { landscapes }\end{array}$ & & & & & 206 & & & & & & 112 & & \\
\hline
\end{tabular}

\section{Dominance structure of wild bees}

In the agricultural landscape of the Pleven region, mostly halictid bees were caught, representing Evylaeus, Halictus, and Lasioglossum species. The dominant taxon was $E$. malachurus, which accounted for $12 \%$ of the total number of bees at that site. It was eudominant at most study sites (12 - 18\% of the total) and ranked second after the honey bee as a major flower visitor of sunflower (over 35\%). It is a eusocial species, with remarkable morphological variation among castes: worker bees ( $F$. longulus) are smaller and have more delicate mandibles than queens. Males emerge at the height of summer. The species prefers open, dry habitats, which also are present in the study area. It is regarded as a major pollinator of alfalfa while in the studied alfalfa seed production field it accounted for $10 \%$ of wild bees.

Halictus compressus/simplex (= eurygnathus/ simplex) are subdominants. These 2 species of the subgenus Monilapis Ckll. - $\mathrm{H}$. simplex (Blüthgen, 1923 ) and H. compressus (Walckenaer, 1802) (= eurygnathus Blüthgen, 1931) - have identical females. Here they jointly constituted almost $9 \%$ of wild bees in that landscape, but only single males were caught.
These species were found mostly on herbaceous vegetation along the ditch in the Pleven region (over $13 \%$ ), between the ditch and the maize field (nearly $12 \%$ ), and in the alfalfa field (nearly 15\%). Both eusocial species nest in dry and open habitats, as this study confirmed.

Relatively frequent ( $46 \%$ of the total catch) were also Andrena flavipes, Halictus maculatus, Rhophitoides canus, and Evylaeus corvinum. Each of them at some study sites reached a high proportion of wild bees. Andrena flavipes was eudominant in the rape field (almost 16\%) and radish field (20\%), and its contribution was considerable (4-6\%) on the roadside, at the ditch edge, and along the ditch near a maize field. Halictus maculatus dominated (over $8 \%)$ in herbaceous vegetation along the ditch near maize (14\%), at the ditch edge (about 9\%), and on roadsides (about 5\%). Rhophitoides canus was an overwhelmingly dominant flower visitor of alfalfa (over 32\%, 315 individuals) in the alfalfa seed plantation, indicating that the habitat was probably a nesting site for this species. 


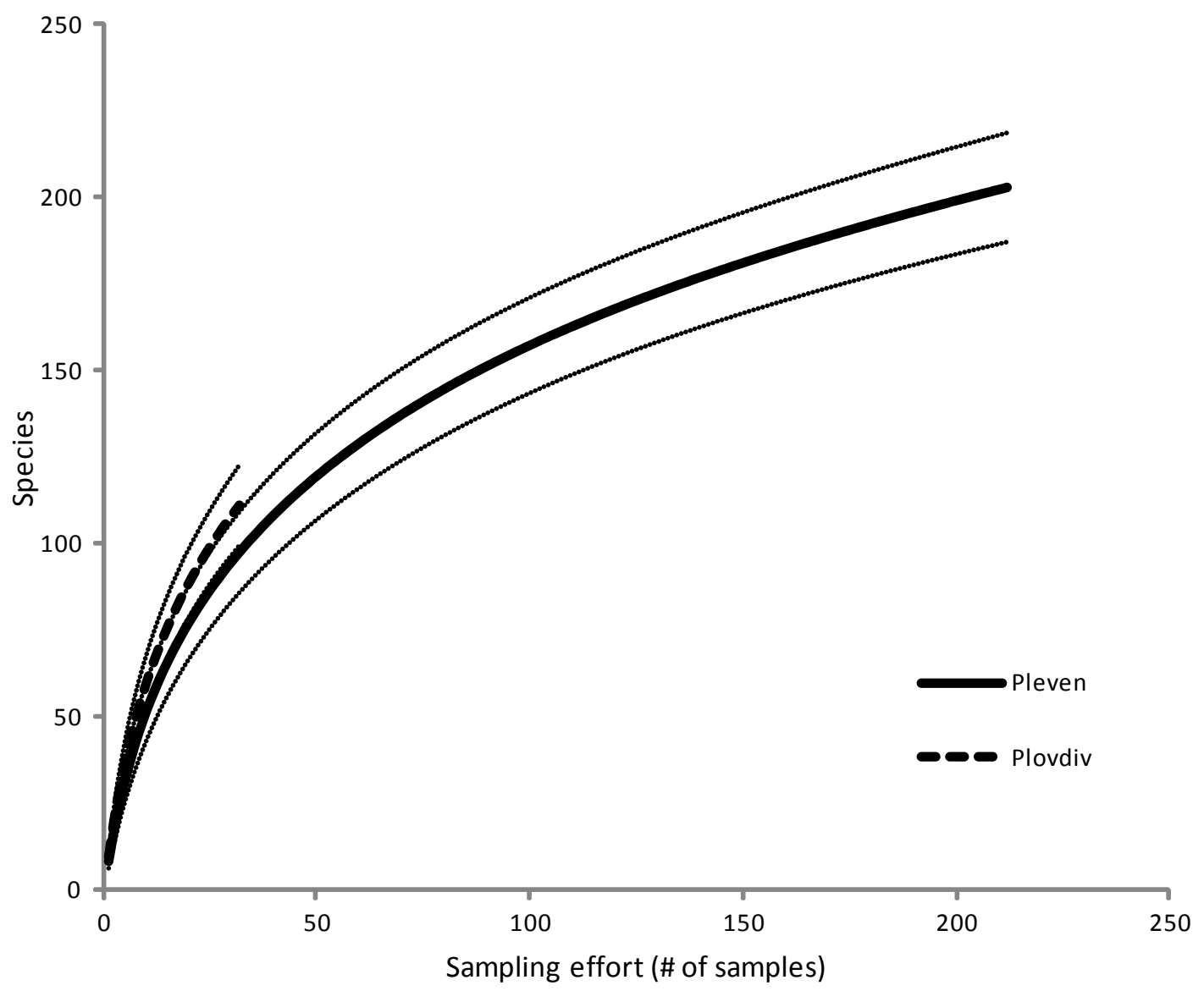

Fig. 1. Expected cumulative number of species (MaoTau estimator) as a function of number of samples for 2 Bulgarian agricultural landscapes. Dotted lines indicate 95\% confidence intervals.

Evylaeus corvinum was a subdominant (about 11\%) in herbaceous vegetation along the ditch and on slopes of the Vit valley near Pleven (over 13\%, beside Evylaeus malachurus) whereas aboutand had a frequency of about 5 - $6 \%$ as a flower visitor of sunflower and oilseed rape. Another noteworthy species was Andrena tringa, which was represented in our material almost exclusively by 55 and 58 individuals caught in fields of rape and radish, respectively. Thus, it must nest within the fields or in the immediate neighborhood because in the other refuge habitats, it was represented by only a single individual on herbaceous vegetation along the ditch. Two years of research at 3 study sites in the agricultural landscape of the Plovdiv region detected a very high contribution of Halictidae, very much like in the lowland near Pleven. The major species was Heriades crenulatus (about 4.2\%) whereas the contribution of Andrena spp. was low. Systropha curvicornis was eudominant (about 22\%) in the grass and shrub communities along railway tracks and dominant $(10 \%)$ at the ditch edge and on the roadside (about $8 \%$ ). This species is common in southern and central Europe to western and central Asia and is oligolectic, associated mostly with Convolvulus. It is recorded in summer, nesting in large aggregations, in nests up to $12-18 \mathrm{~cm}$ deep. Eudominants included also Halictus resurgens (about $12 \%$ ), which was most numerous at the edge of the ditch (about 19\%) and on the roadside (over 13\%). Another frequent species was Evylaeus truncaticollis (about 9\%), which was eudominant on the roadside (over $18 \%$ ).

In comparisons of dominance structure (rank-abundance) (Fig. 3), the Student's t test showed a significant difference $(p=0.02)$ between $E_{Q}$ values for crop fields (mean 0.2684) and refuge habitats (mean 0.3159 ). Lower values for fields were associated mostly with low species number and their high dominance in the community, as was confirmed by higher negative values of slope angle for most frequent species in fields compared to semi-natural habitats (means -4.57883 and -3.12336 , respectively; Student's t test: $p<0.001)$. Higher $E_{Q}$ values for bee communities of refuge habitats resulted from more even division of environmental resources 
1987

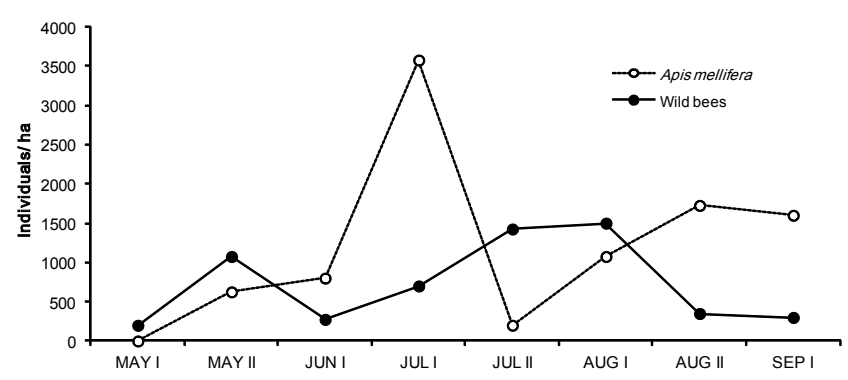

1988

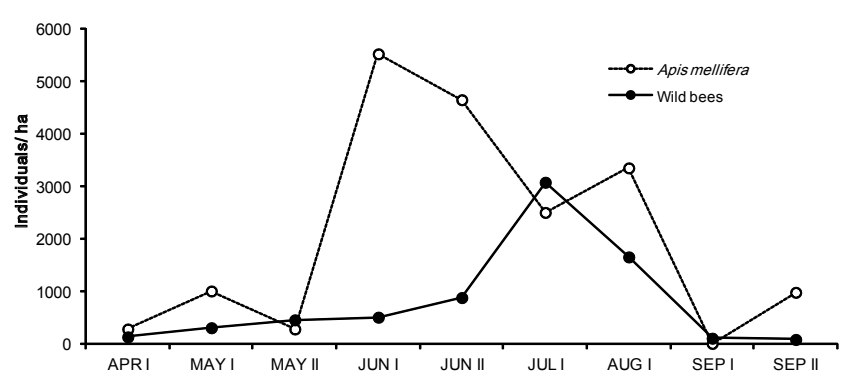

Pleven-Brassica napus v. oleifera „Elena" , 1990

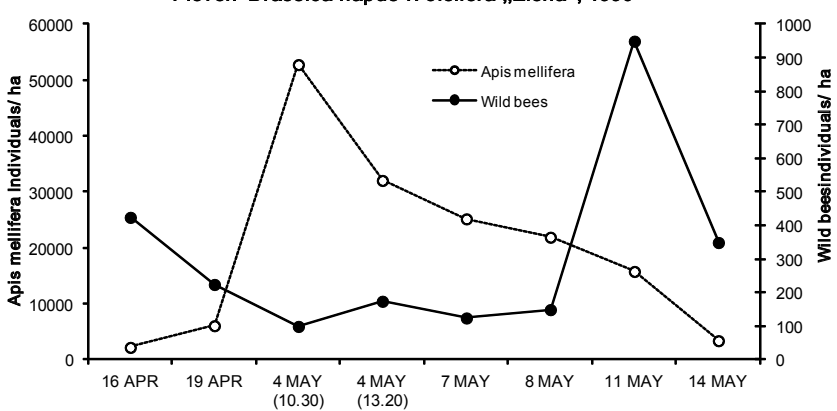

1987

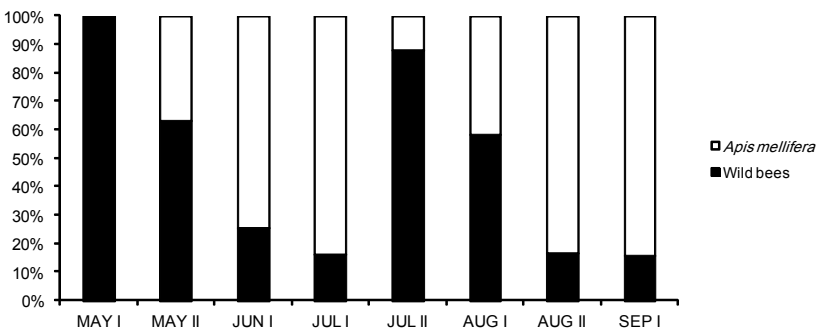

1988

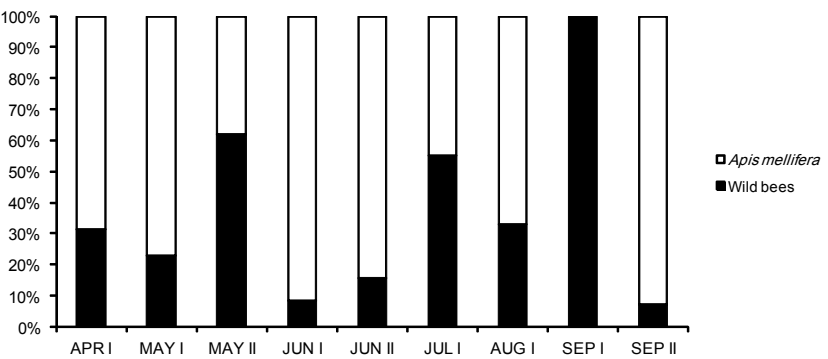

Pleven-Brassica napus v。 oleifera "Elena", 1990

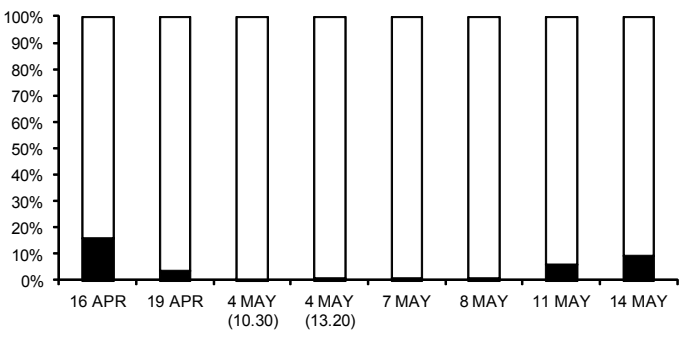

- Apismellifera Wild bees

Fig. 2. Examples of changes in abundance and relative contributions of Apis mellifera and wild bees in a refuge habitat (ditch edge) in 1987 and 1988, and in an oilseed rape field

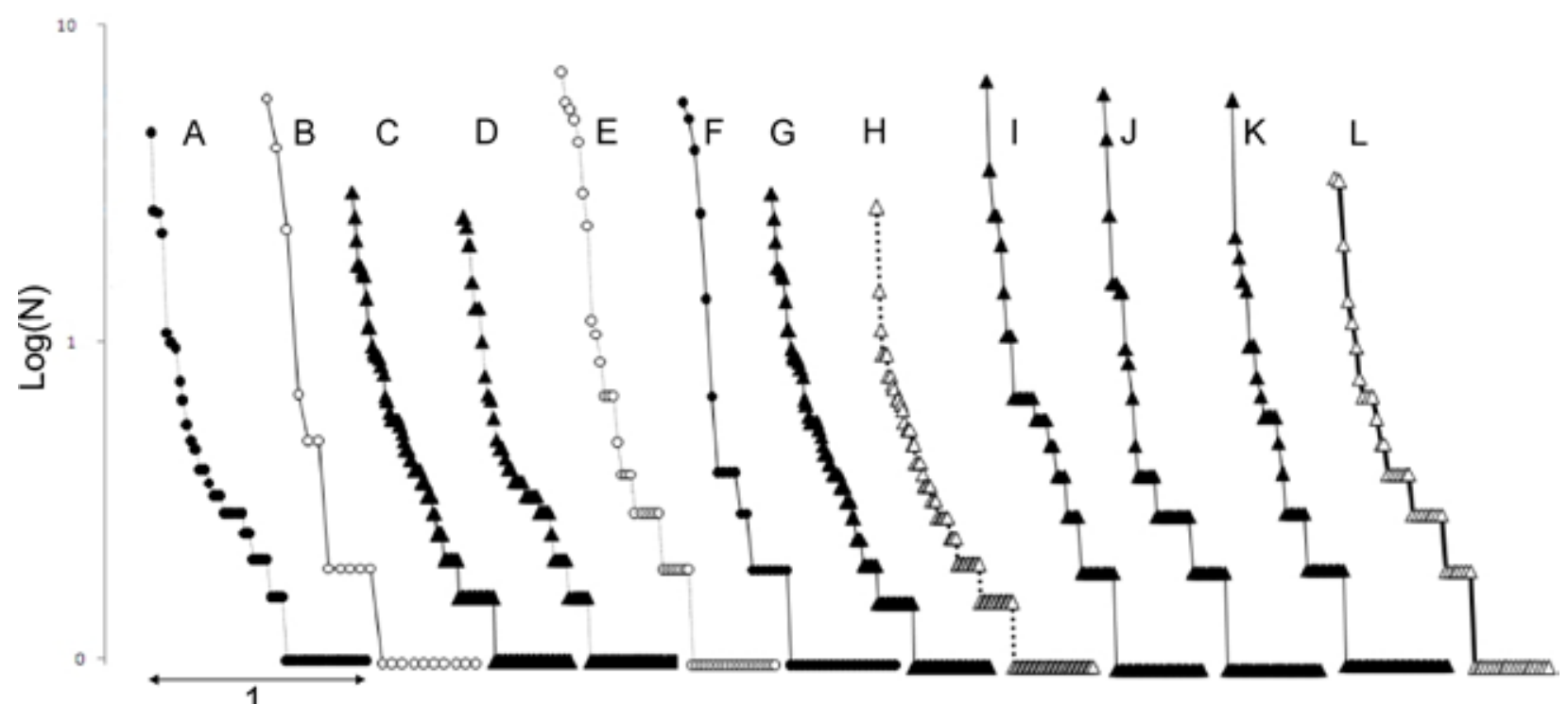

Fig. 3. Rank-abundance curves for wild bee communities in the agricultural landscape of Pleven $(A-H, L)$ and Plovdiv $(\mathrm{I}-\mathrm{K})$. For comparison, the ranks were scaled to range from 0 to 1 . The curves are in ascending order of $E Q$ values. A - Alfalfa field, B - Sunflower field, C - Roadside, D - Ditch near maize, E - Rape field, F - Radish field, G - Ditch edge, $\mathrm{H}$ - Vit river valley, I - Ditch edge (Plovdiv), J - Roadside (Plovdiv), K - Railway embankment, L - Slope of Vit valley. 


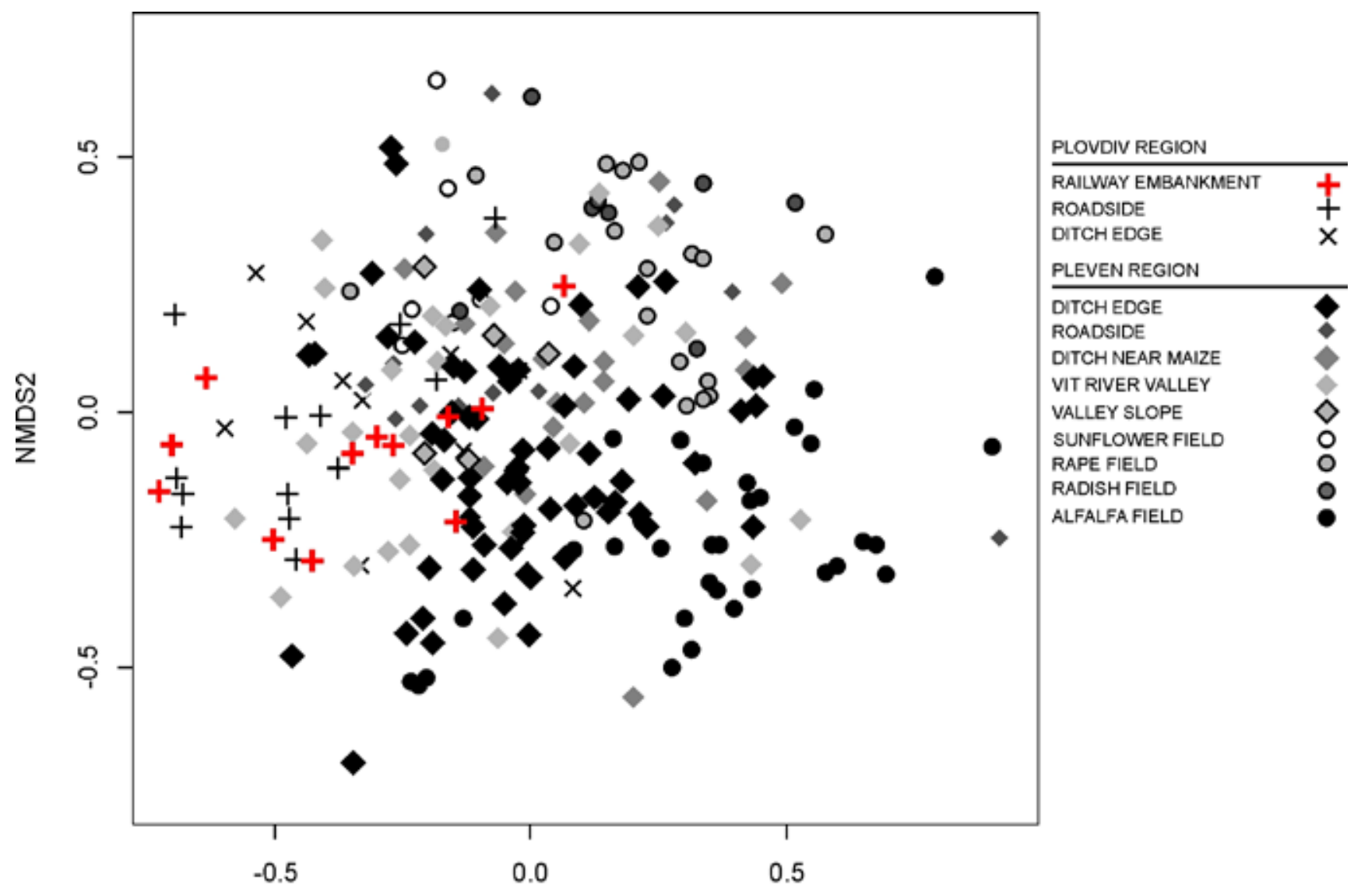

NMDS1

Fig. 4. NMDS ordination diagram of bee communities in habitats of the Pleven and Plovdiv regions based on the whole material. Each point on the diagram represents a single sample (231 for both crop fields (71) and semi-natural habitats (160) from the Pleven region; 38 from the Plovdiv region). Distances between them reflect similarity between bee communities in both abundance and diversity based on the Bray-Curtis dissimilarity index.

among bee species. These values were also reflected in the greater slope angle for the most frequent species in the community, i.e., smaller contributions of most frequent species.

Substantial differences in bee community structure and composition between semi-natural habitats of the 2 landscapes were confirmed by the results of NMDS (Fig. 4). Samples collected from semi-natural habitats of the agricultural landscape of Pleven are located mostly in the central part of the NMDS ordination diagram while samples collected from 3 sites in the Plovdiv region are in the left part. The multi-response permutation procedure confirmed significant differences between wild bee communities of the 2 regions ( $p<0.001, A=0.0217$ ), but at the same time confirmed a relatively low homogeneity of samples within the 2 groups of sites.

The analyzed field habitats clearly differed in bee community structure. The calculated statisticalIy significant IndVals for species (Tab. 2) made it possible to identify which of them are responsible for the observed differences. The analysis showed that different species were characteristic for the compared types of agricultural landscapes. The Pleven region was characterised by the presence of Evylaeus malachurus and Halictus simplex/eurygnathus. Species with lower IndVals were Andrena flavipes and Halictus quadricinctus. The bee community of the Plovdiv region was distinguished primarily by the presence of Evylaeus lucidulus, Systropha curvicornis, and Halictus resurgens. Additionally, E. truncaticolle showed a lower fidelity and constancy in samples.

\section{DISCUSSION}

Bulgaria lies along the borders of several zoogeographic regions, near the Mediterranean basin, Black Sea, and Central Europe. Its location undoubtedly is one reason for its great diversity of animals, including bees, although the latter remain poorly studied. There is little published information on the diversity of Apiformes in this country, with no documented list of bee species and not even any estimates. 
Table 2.

Calculated significant indicator values for species in the 2 analyzed landscapes

\begin{tabular}{cccc}
\hline Landscape & Bee species & $\begin{array}{c}\text { Indicator } \\
\text { value }\end{array}$ & Probability \\
\hline \multirow{3}{*}{$\begin{array}{c}\text { Pleven } \\
\text { region }\end{array}$} & Evylaeus malachurus (Kirby, 1802) & 0.47 & 0.004 \\
\cline { 2 - 4 } & Halictus simplex/compressus & 0.43 & 0.011 \\
\cline { 2 - 4 } & Andrena flavipes Panzer, 1799 & 0.28 & 0.032 \\
\cline { 2 - 4 } & Halictus quadricinctus (Fabricius, 1776) & 0.25 & 0.001 \\
\cline { 2 - 4 } & Evylaeus corvinum (Morawitz, 1878) & 0.24 & 0.015 \\
\cline { 2 - 4 } Plovdiv & Evylaeus lucidulus (Schenck, 1861) & 0.47 & 0.001 \\
\cline { 2 - 4 } region & Systropha curvicornis (Scopoli, 1770) & 0.46 & 0.001 \\
\cline { 2 - 4 } & Halictus resurgens Nurse, 1903 & 0.38 & 0.003 \\
\cline { 2 - 4 } & Evylaeus truncaticolle Morawitz, 1887 & 0.29 & 0.007 \\
\cline { 2 - 4 } & Evylaeus interruptum (Panzer, 1798) & 0.24 & 0.001 \\
\cline { 2 - 4 } & Heriades crenulatus Nylander, 1856 & 0.24 & 0.001 \\
\hline
\end{tabular}

Atanassov (1972a, b) in the Balkan Mountains (Stara Planina) found 189 species of Apiformes. From the Petrič region, he reported 119 species (Atanassov, 1965), and from the island of Tasos, 27 species (Atanasov, 1965). Dimitrov (1987) and Dimitrov et al. (1987) near the city of Ruse (also known as Russe or Rousse) listed 40 - 51 bee species from an alfalfa field. Atanassov's (1960) study on the Halictidae brought information about 60 species, which certainly does not reflect the true number of species of this family. Dochkova et al. (1987) in an earlier study of the Pleven region, listed 85 species collected in fields of Medicago sativa, Trifolium pratense, Lotus corniculatus, and Onobrychis sativa. In the present work, data from the Pleven region have provided information about the occurrence of 204 species but did not confirm 44 species reported earlier by the cited authors. Overall, with the current investigation, the total number of bee species recorded in the Pleven region has increased to 248 species.

This short review of available data shows that the current knowledge of the bee fauna of Bulgaria concerns only a few local fauna with respect to natural habitats, as in the case of Atanasov's work. So far, the typically poorer agricultural landscape has been studied more extensively, although still at an initial stage. Predictions about true species richness concerning Pleven and Plovdiv indicate that up to 300 bee species can be expected.

It is also interesting to compare percentage contributions of individual bee families to the apifauna of various agricultural landscapes in 3 countries: Bulgaria, Romania, and Poland. Contributions of major families are similar, especially of the Andrenidae, Halictidae, Megachilidae, and Anthophoridae (Fig. 5). By contrast, there are large differences in contributions of the Colletidae and Apidae to the fauna of the compared landscapes.

Some comments about bumblebees must be added. Their species diversity was very low; they were represented in this study by only 11 species in both lowlands (the other 4 were found during occasional sampling near Sofia), and their abundance was also extremely low. So far, a total of 29 species of Bombus have been found in Bulgaria (Pittioni, 1938; Atanassov, 1939). In both lowlands, we caught only 337 specimens of bumblebees, which accounts for only $4.8 \%$ of the total. However, they were even less numerous in the agricultural landscape of Romania, where only 8 species were recorded (Banaszak and Manole, 1987). This information indicates that bumblebees play a minor role in the farmland of the lowland part of the country, as confirmed by the negligible density of these insects in the investigated fields, especially in the sunflower field. Research on pollinators of this plant, conducted by Dimitrov et al. (1992) in north-eastern Bulgaria, showed that bumblebees accounted for $20 \%$ while honey bees accounted for $62 \%$. In Romania in sunflower fields, honey bees pollinated almost $100 \%$ of flower heads (Banaszak and Manole, 1987) whereas in Poland, bumblebees accounted for 30\% (Banaszak, 1984). The low number of species of bumblebees and their low abundance in South European lowlands can be explained by the association of these insects with cooler climates, which has been reported previously 


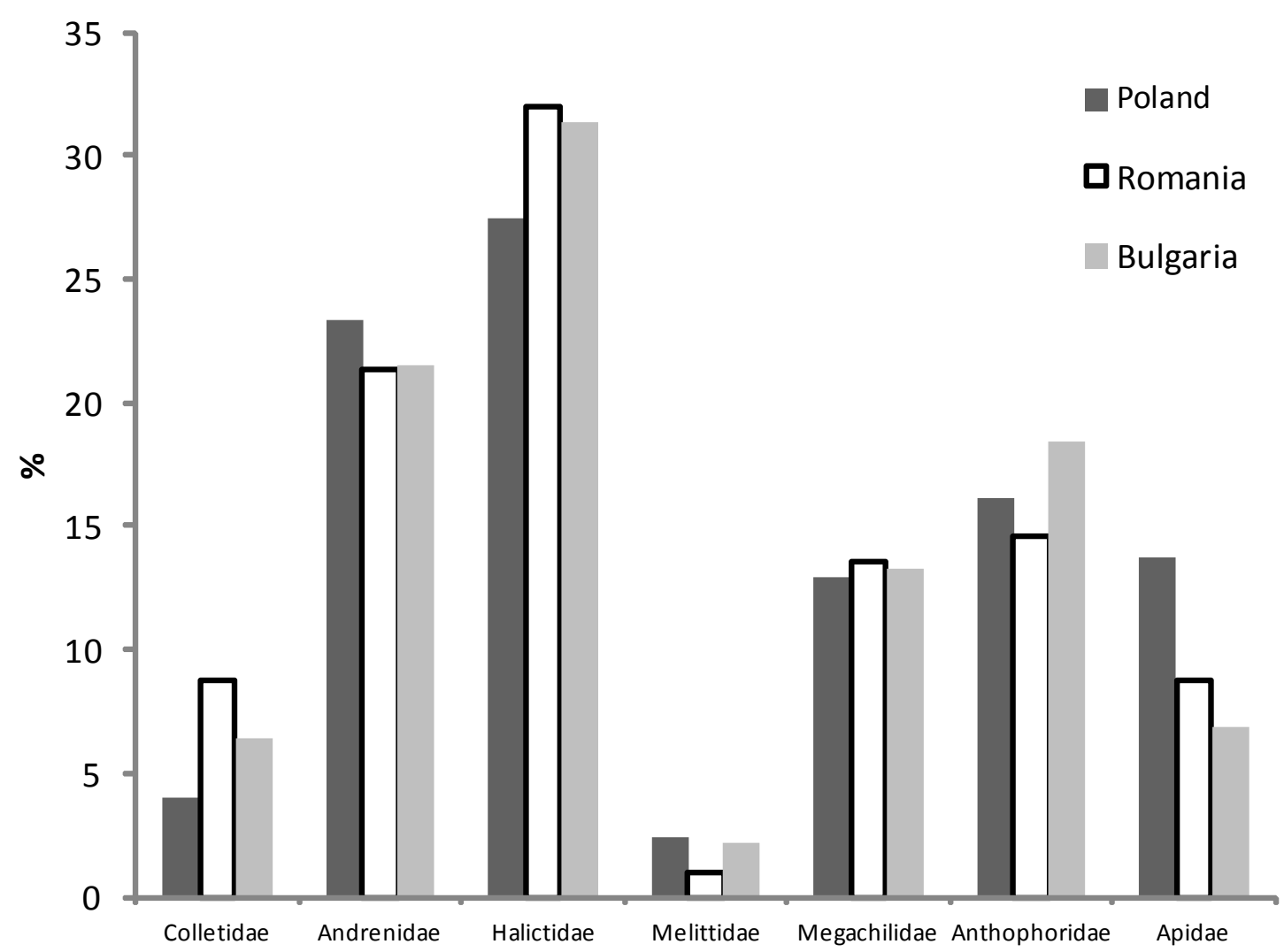

Fig. 5. Comparison of relative contributions of families of Apiformes in agricultural landscapes of Poland (Banaszak, 1983, 1984; Banaszak and Cierzniak, 1998), Romania (Banaszak and Manole, 1987), and Bulgaria.

(Banaszak, 1996). Most bumblebee species reported from Bulgaria live in submontane areas and in mountains (Atanassov, 1939).

Of note, in both investigated agricultural landscapes, the collected bees included very few or no parasitic species. In the study area, we found no Psithyrus spp., although at least 8 species exist in Bulgaria (Atanassov, 1939, 1974). We recorded only 7 species of Sphecodes and 11 of Nomada. These findings confirm that parasitic species are found mostly in permanent ecosystems whereas agroecosystems and the associated field margins are temporary ecosystems. It is assumed that in permanent communities, the contribution of parasites is higher (Archer, 1995; Calabuig, 2000). A lack or low contribution of parasites to the bee community indicates that the population of the host species is unstablei.e., that the host species has recently colonized the given site-or that its population size varies greatly between years, or that occurrence of the species is ephemeral and does not result from nesting at the site. This finding is consistent with reports of Banaszak (1983), Cierzniak (2003), and BanaszakCibicka and Żmihorski (2012).
Among the investigated crop plant species, alfalfa pollinators are best studied. Research carried out by Dochkova et al. (1981a) in the Pleven region supplied information on the occurrence of 65 wild bee species; research by Dimitrov et al. (1987) near the city of Ruse detected 51 species. A comparable number (54 species) is reported in the present study. All the cited authors and the present study show that the major pollinators of alfalfa are Melitta leporina, Eucera clypeata, and Rhophitoides canus. According to Dochkova et al. (1981a), $R$. canus was infrequent in 1978 - 1979. In the Pleven region, they observed a high abundance of Melitturga clavicornis, Megachile pilidens, Eucera longicornis, and $E$. interrupta. Dimitrov et al. (1987) in the Ruse region also recorded a high contribution of Bombus sylvarum. These differences probably result mostly from the time of insect collection, i.e., the flowering period (there are 3 possible flowering periods of alfalfa). 


\section{CONCLUSIONS}

The present studies of bees (Apiformes) in the agricultural landscape of Bulgaria showed the presence of 206 species in the Danubian Plain (the Pleven region) and 112 species in the Upper Thracian Plain (the Plovdiv region) (Tab. 1). The total of 7046 specimens of 232 wild bees species were collected. Regardless to the differences in the found numbers of species resulting from the different collection periods, the rarefaction curves calculated for both landscapes indicated similar diversity and species richness.

The Pleven region was characterised by the presence of Evylaeus malachurus and Halictus simplex/ eurygnathus. Eusocial Evylaeus malachurus turned out to be the main, after the honey bee, pollinator of sunflower. The following species were weaker indicators: Andrena flavipes and Halictus quadricinctus. The communities of the Plovdiv region bees were strongly characterised by Systropha curvicornis, Evylaeus lucidulus and Halictus resurgens.

In both studied lowlands, low species diversity and especially low abundance of bumblebees can be noticed. This can be explained by the fact that these species prefer cooler and more humid climate.

Among the studied crops the composition of pollinators of alfalfa is examined to the broadest extent. 54 visiting species were found on this plant species with Rhophitoides canus, Melitta leporina and Eucera clypeata being the most abundant.

Both plains were characterised by low number or lack of parasitic species.

Apis mellifera was overwhelmingly dominant (80 - 100\%) pollinator of the studied plantations, with relatively small (at most $20 \%$ on rapeseed) share of wild bees.

\section{ACKNOWLEDGEMENTS}

We are grateful to P. Andreas Werner Ebmer (Pfarer von Puchenau near Linz, Austria) for identification or verification of several dozen difficult species of the family Halictidae. Moreover, we owe our gratitude to Prof. Halina Ratyńska (Kazimierz Wielki University of Bydgoszcz, Poland) for botanical consultations. Sincere thanks are also due to assistants of the first author, Piotr Szefer, MSc, and Olga Sondej, MSc, for help in statistical and technical processing of the data. We are also grateful to Sylwia Ufnalska, MSc, MA, who translated this paper into English.

\section{REFERENCES}

Archer M. E. (1995) Aculeate wasps and bees Hymenoptera: Aculeata of Blaxton Common in Watsonian Yorkshire with the introduction of a new national quality scoring system. Naturalist 120(1012): 21-29.

Atanassov N. (1939) Beitrag zum Studium der Hummelfauna Bulgariens (Bombus - Hymenoptera). Mitteilungen der Bulgarischen Entomologishen Gessellschaft in Sofia 10: 91-109.

Atanassov N. (1960) Untersuchungen über die Arten aus der Familie Halictidae (Hymenoptera) in Bulgariens und der Balkanhalbinsel. Izvestiya na Zoologicheskiya institut s muzei 9: 315-338.

Atanassov N. (1962a) Untersuchungen über die Arten von der Gattung Xylocopa Latr. (Hymenoptera, Apoidea) in Bulgarien und auf der Balkanhalbinsel. Bulletin de l'Institut de zoologie et musée 1 1: 167-180.

Atanassov N. (1962b) Untersuchungen über die systematik und die Ökologie der Hymenopterenarten aus dem Gebiet von Petrič (Südwestbulgarien). Izvestiya na Zoologicheskiya institut s muzei 12: 109-172.

Atanassov N. (1965) Hymenoptera von der Insel Thasos. Bulletin de l'Institut de zoologie et musée 19: 85-99.

Atanassov N. (1972a) Hymenopteraarten im Westlichen „Stara Planina" - Gebirge. I. Bulletin de l'Institut de zoologie et musée 35: 179-228.

Atanassov N. (1972b) Hymenopteraarten im Westlichen "Stara Planina" - Gebirge. II. Bulletin de l'Institut de zoologie et musée 36: 23-59.

Atanassov N. (1 974) Hummeln und Schmarotzerhummeln (Bombus Latr., Psithyrus Lep., Hym.) von dem Mittleren und dem Östlichen Balkangebirge. Bulletin de l'Institut de zoologie et musée 41: 107-121.

Banaszak J. (1980) Studies on methods of censusing the numbers of bees (Hymenoptera, Apoidea). Polish Ecological Studies 6(2): 355-366.

Banaszak J. (1983) Ecology of bees (Apoidea) of agricultural landscape. Polish Ecological Studies 9(4): 421-505.

Banaszak J. (1984) Występowanie i zagęszczenie pszczół (Apoidea) na plantacjach wybranych roślin uprawnych w Wielkopolsce. Polskie Pismo Entomologiczne 53: 623631. 
Banaszak J. (1996) Variability in density of bumblebees in Europe (Hymenoptera: Apoidea: Bombus Latr.). Polish Journal of Entomology 65(1-2): 21-31.

Banaszak J., Cierzniak T. (1998) Owady zapylające - Apoidea. In: Banaszak J. (Ed.) Ekologia wysp leśnych. WSP University Press. Bydgoszcz: 113-139.

Banaszak J., Manole T. (1987) Diversity and density of pollinating insects (Apoidea) in the agricultural landscape of Rumania. Polish Journal of Entomology 57(4): 747-766.

Banaszak-Cibicka W., Żmihorski M. (2012) Wild bees along an urban gradient: winners and losers. Journal of Insect Conservation 16(3): 331-343.

Bonner J. L., Anderson J. T., Rentch J. S., Grafon W. N. (2009) Vegetative composition and community structure associated with beaver ponds in Canaan valley, West Virginia, USA. Wetlands Ecology and Management 17(5): 543554.

Burnham, K. P., Overton, W. S. (1979) Robust estimation of population size when capture probabilities vary among animals. Ecology 60(5): 927-936.

Calabuig I. (2000) Solitary bees and bumble bees in a Danish Agricultural landscape. University of Copenhagen. Copenhagen. 240 pp.

Calvillo L. M., Ramírez V. M., Parra-Tabla V., Navarro J. (2010) Bee diversity in a fragmented landscape of the Mexican neotropic. Journal of Insect Conservation 14(4): 323-334. DOI: 10.1007/s10841-010-9262-x

Chao A. (1 984) Non-parametric estimation of the number of classes in a population. Scandinavian Journal of Statistics 11(4): 265-270.

Cierzniak T. (2003) Ekologia pszczół w dynamicznym kręgu zbiorowisk grądowych. Bydgoszcz University Press. Bydgoszcz. 158 pp.

Colwell R. K. (2006) EstimateS: Statistical estimation of species richness and shared species from sample. Version 8.0.0. User's Guide and applications. Available at: http:// purl.oclc.org/estimates

Colwell R. K., Coddington J. A. (1994) Estimating terrestrial biodiversity trough extrapolation. Philosophical Transactions of the Royal Society of London. Series B: Biological Sciences 345(131 1): 101-1 18. D0l: 10.1098/ rstb.1994.0091
Dimitrov P. (1984) Some problems of the scientific and research workin this country associated with the providing of maximum pollination and fertilization of alfalfa (Medicago sativa L.). Agricultural Science 22(3): 93-97.

Dimitrov P. (1987) Studies of species composition and numbers of bee - pollinators (Hymenoptera, Apoidae) of alfalfa according to regrowths. Plant Science 24(1 1): 75 80.

Dimitrov P. (1990) Effect of the density of the wild bees (Hymenoptera, Apoidea) on the percentage of pollination of lucerne blossoms. Plant Science 27(6): 23-27.

Dimitrov P. (1992a) Effect of air temperature on the dynamics of the flight of wild bees (Hymenoptera, Apoidea), lucerne pollinators. Plant Science 29(1-2): 106-111.

Dimitrov P. (1992b) Effect of relative air humidity on the flight of wild bees (Hymenoptera, Apoidea) - Iucerne pollinators. Plant Science 29(3-4): 114-117.

Dimitrov P., Dimitrova Z. (1991a) Studies of the provision of alfalfa fields for seedproduction with pollinating bees (Hymenoptera Apoidea) in conditions of north - eastern Bulgaria. Agricultural Science 29(4-6): 90-93.

Dimitrov P., Dimitrova Z. (1 991b) A rapid method of determining the density of wild bees (Hymenoptera, Apoidea) in lucerne grown for seed. Plant Science 28(3-6): 118 119.

Dimitrov P., Dimitrova Z., Atanassov N., Vasileva E. (1987) Specific body and number of bees (Hymenoptera, Apoidea) polling alfalfa in the region of Rousse district. Plant Science 24(3): 58-62.

Dimitrov P., Dimitrova Z., Piskov A. (1992) Studies of beepollinators (Hymenoptera Apoidea) in sunflower hybrid seedproduction. Agricultural Science 30(1-3): 22-26.

Dochkova B. (1981a) Alfalfa Megachile rotundata F. (= pacifica Pz.) (Hymenoptera, Megachilidae). I. Morphological and biological characteristics. Plant Science 18(8): 91-98.

Dochkova B. (1981b) Bees-pollinators (Hymenoptera, Apoidea) of alfalfa in the region of Pleven. V. Economic importance. Plant Science 18(5): 148-155.

Dochkova B. (1982a) Alfalfa leaf- cutting bee Megachile rotundata F. (= pacifica Pz.) (Hymenoptera, Megachilidae). II. Building and pollination activity. Plant Science 19(1): 112-118. 
Dochkova B. (1982b) The alfalfa leaf cutter-bee (Megachile rotundata F.) (= pacifica Pz.) (Hymenoptera, Megachilidae). III. Natural pests and possibilities for their control. Plant Science 19(2): 1 18-127.

Dochkova B. (1984) Studies of the economic importance of Megachile rotundata F. (Hymenoptera, Megachilidae). Plant Science 21(4): 116-121.

Dochkova B., Atanassov N., Atanassova E. (1984) Morpho-Ecological Studies on Halictus calceatus Scop. (Hymenoptera, Halictidae). Ekologiia 3(13): 56-65.

Dochkova B., Atanassov N., Vassileva E. (1981a) Alfalfa pollinating bees (Hymenoptera, Apoidea) in the region of Pleven. I. Species composition and numbers. Plant Science 18(1): 102-108.

Dochkova B., Atanassov N., Vassileva E. (1981b) Bees pollinators (Hymenoptera, Apoidea) of alfalfa in the region of Pleven. II. Nutrient specialization. Plant Science 18(2): 97-102.

Dochkova B., Atanassov N., Vassileva E. (1981c) Bees - pollinators (Hymenoptera, Apoidea) of alfalfa in the region of Pleven. III. Seasonal dynamics of the species. Plant Science 18(3): 102-109.

Dochkova B., Atanassov N., Vassileva E. (1981d) Bees - pollinators (Hymenoptera, Apoidea) of alfalfa in the region of Pleven. IV. Rate of pollination. Plant Science 18(4): 140-146.

Dochkova B., Atanassov N., Vassileva E. (1987) Species composition and number of the wild bees (Hymenoptera, Apoidea) - pollinating on the forage loants from the region of Pleven. Acta Zoologica Bulgarica 35: 34-43.

Dufrêne M., Legendre P. (1997) Species assemblages and indicator species: the need for a flexible asymmetrical approach. Ecological Monographs 67(3): 345-366. DOl: 10.1890/0012-9615(1997)067[0345:SAAIST]2. $0 . \mathrm{CO}, 2$

Faith D. P., Minchin P. R., Belbin L. (1987) Compositional dissimilarity as a robust measure of ecological distance. Vegetatio 69(1-3): 57-68.

Gotelli N. J., Colwell R. K. (2001) Quantifying biodiversity: procedures and pitfalls in measurment and comparison of species richness. Ecology Letters 4(4): 379-391.
Gotelli N. J., Colwell R. K. (2010) Estimating species richness. In: Magurran A. E., McGill B. J. (Eds.) Biological Diversity: Frontiers In Measurement And Assessment. Oxford University Press. Oxford: 39-54.

Hannon L. E., Sisk T. D. (2009) Hedgerows in an agrinatural landscape: Potential habitat value for native bees. Biological Conservation 142(10): 2140-2154. DOl: 10.1016/j.biocon.2009.04.014

Oksanen J., Blanchet F. G., Kindt R., Legendre P., O'Hara R. B., Simpson G. L., Solymos P., Stevens M. H. H., Wagner H. (201 1) Vegan: Community Ecology Package. R package version 1.17-1 1. Available at: http://CRAN.R-project.org/ package=vegan

Pittioni B. (1938) Die Hummeln und Schmarotzerhummeln der Balkan-Halbinsel. Mitteilungen aus den Koniglischen naturwissenschaftlichen Instituten in Sofia 11:12-69.

Pittioni B. (1939) Die Hummeln und Schmarotzerhummeln der Balkan-Halbinsel. II. Mitteilungen aus den Koniglischen naturwissenschaftlichen Instituten in Sofia 12: 49-115.

R Development Core Team (2008) R: A language and environment for statistical computing. R Foundation for Statistical Computing. Vienna. Available at: http:// www.R-project.org.

Roberts D. W. (2010) Labdsv: Ordination and Multivariate Analysis for Ecology. R package version 1.4-1. Available at: http://CRAN.R-project.org/package=labdsv

Smith B., Wilson J. B. (1996) A Consumer's Guide to Evenness Indices. Oikos 76 (1): 70-82.

Zimmerman G. M., Goetz H., Mielke Jr. P. W. (1985) Use of an improved statistical method for group comparisons to study effects of prairie fire. Ecology 66(2): 606-61 1.

Żmihorski M., Sienkiewicz P., Tryjanowski P.. (2013) Never ending story: a lesson in using sampling efficiency methods with ground beetles. Journal of Insect Conservation 179(2): 333-337. DOl: 10.1007/s10841-012-9514-2 